\title{
Cinema per i non vedenti. Dispositivi tattili per la fruizione
}

\author{
Daniele Colistra \\ Giada Puccinelli
}

Abstract

Questo studio presenta i primi risultati di una ricerca sul tema dei dispositivi tattili per favorire la fruizione dei film da parte dei non vedenti. Un argomento allo stato attuale poco indagato, i cui principi non sono ancora stati sistematizzati e che, pertanto, offre ampi margini di sperimentazione. La ricerca è ancora in corso ed è stata contemporaneamente sviluppata per la fotografia e le arti grafiche bidimensionali. In questa sede ci limiteremo a trattare la cinematografia, pur facendo riferimento a considerazioni che, in buona parte, sono valide anche per altre forme figurative.

Gli strumenti ausiliari attualmente a disposizione di un non vedente per la fruizione di un film consistono esclusivamente in supporti audio che integrano i dialoghi e offrono informazioni minime su elementi visuali rilevanti per lo sviluppo della narrazione. Le integrazioni audio, basate sulla descrizione verbale, non sono però idonee a rendere molte componenti espressive legate alla visualità (inquadrature, ottiche, movimenti di macchina, scelte legate al montaggio ecc.) che, com'è evidente, costituiscono elementi imprescindibili per apprezzare pienamente un film.

Gli studi ormai avanzati e i primi prototipi realizzati nel campo dei dispositivi tattili digitali (tablet a microsfere motorizzate), anche se ancora non disponibili su scala commerciale, potrebbero ampliare un settore economicamente rilevante e di grande interesse scientifico. Inoltre, permetterebbero l'inclusività e un maggiore accesso alla cultura da parte di una categoria svantaggiata. In questo saggio descriveremo il metodo sviluppato per realizzare i supporti tattili delle scene chiave di un film, applicandolo a La finestra sul cortile (RearWindow) di Alfred Hitchcock e successivamente valutando i limiti e le potenzialità della sperimentazione.

Parole chiave

cinema per non vedenti, aptica, sussidi tattili, disegno in rilievo, La finestra sul cortile.

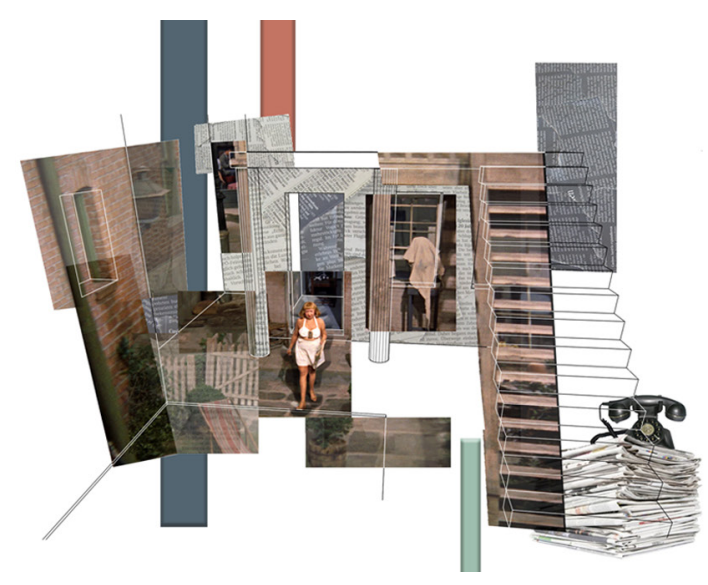

3129

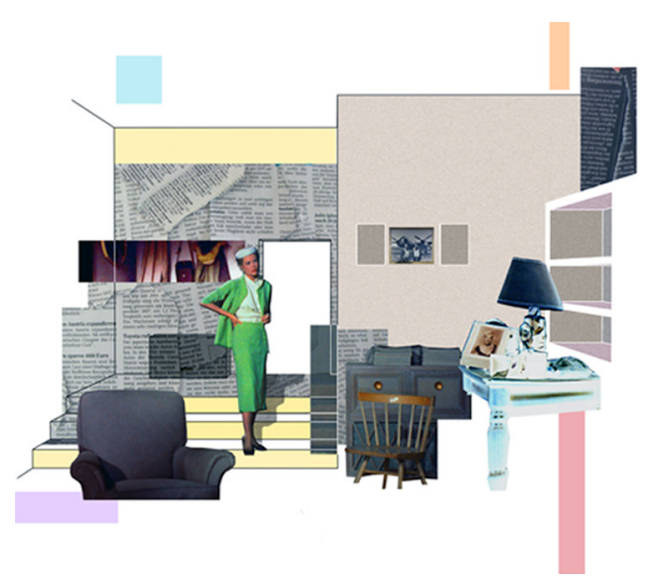

doi.org/10.3280/oa-548.17| 


\section{Premessa e stato dell'arte}

Un cieco ha la perfetta consapevolezza cinestetica di un ambiente a lui noto; è in grado di muoversi a suo agio nello spazio e riesce a relazionarsi ad esso con il proprio corpo. Attraverso il tatto, i ciechi acquisiscono in modo analitico un gran numero di informazioni che però, durante la fase aptica, sono disgiunte da uno schema di riferimento tridimensionale unitario. Tramite la vista, i vedenti percepiscono gli oggetti convessi (per esempio: un vaso di ceramica) e gli spazi concavi (per esempio: l'interno di una chiesa) innanzitutto in modo generale e sintetico; poi, eventualmente, in modo analitico. Per i ciechi il procedimento è inverso: gli oggetti e gli spazi vengono ricostruiti sommando le informazioni acquisite, per parti disgiunte, attraverso il tatto o il movimento all'interno di essi. La comunicazione verbale scritta per i non vedenti, com'è noto, si basa su un codice (il Braille) che riproduce tutti i segni fonetici presenti nella scrittura a stampa e, quindi, la trasmissione delle informazioni avviene senza alcuna perdita di significato. Per questo motivo, le modalità di comunicazione coi non vedenti e fra i non vedenti hanno sempre privilegiato le forme verbali, scritte o sonore, evitando il più possibile il ricorso a informazioni di tipo spaziale, cromatico o comunque riconducibili alla sfera visiva [I].

Anche gli strumenti attualmente disponibili per favorire la fruizione di un film si basano su supporti audio integrativi [2]: una voce narrante descrive sommariamente la scena, i gesti e le azioni, in alcuni casi i movimenti della macchina da presa o le scelte relative al montaggio; restano tuttavia escluse numerose qualità, prevalentemente visuali, che sono alla base della cinematografia e che dovrebbero essere trasmesse, pur se in modo parziale. Attraverso le integrazioni audio, il cieco potrà comprendere meglio il film ma non potrà cogliere e apprezzare moltissime delle sue qualità intrinseche, legate appunto alla visualità.

Perché, a differenza dei supporti per la fotografia e la pittura [3], non si è riscontrato un analogo sviluppo in campo cinematografico? Principalmente per due motivi: innanzitutto perché un cieco è comunque in grado di 'comprendere' e in parte apprezzare il film anche solo tramite l'udito; poi perché le numerosissime immagini che compongono un lungometraggio impongono la realizzazione di un numero elevato di dispositivi tattili che, comunque, sarebbero adatti solo per riprodurre le scene statiche, prive di movimenti di macchina e di azioni rapide. I recenti annunci relativi alla produzione in serie di un tablet a rilievo interattivo, adatto non solo per la scrittura Braille ma anche per la percezione di forme complesse, potrebbe far superare rapidamente le attuali e apparentemente insormontabili difficoltà [4].

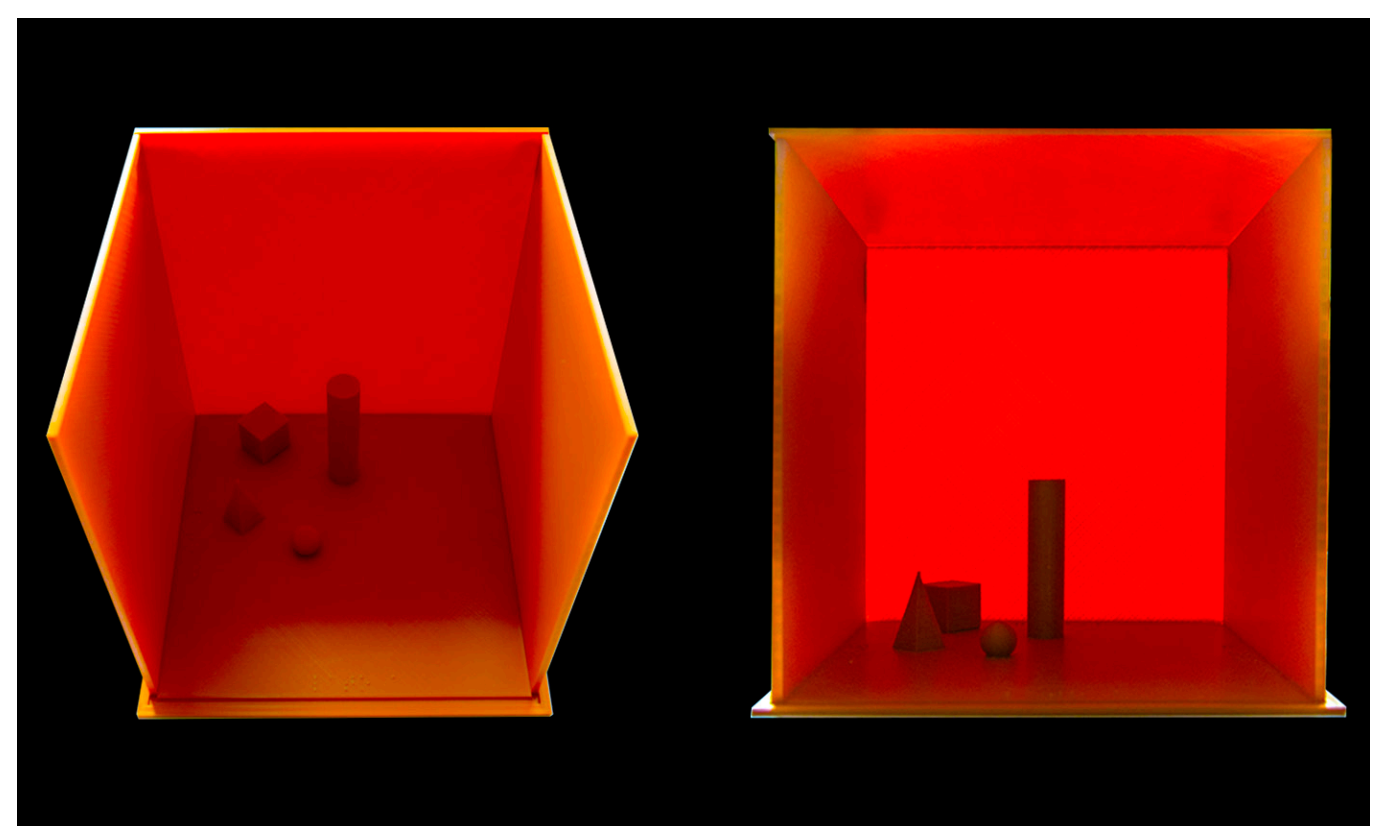




\section{Aptica e propriocezione. Un modello sperimentale per la percezione spaziale}

Il primo problema che abbiamo affrontato consiste nella rappresentazione unitaria di uno spazio concavo. I vedenti percepiscono lo spazio attraverso immagini che si formano sulla retina; la prospettiva lineare è la forma della rappresentazione più analoga a ciò che vediamo con gli occhi. Ma essa stessa è dotata di elementi di convenzionalità, simbolicità [5] e, quindi, necessita di chiavi di lettura per la sua corretta interpretazione. Abbiamo provato a definire un codice elementare per la comunicazione dello spazio tridimensionale tramite un supporto tattile (bassorilievo) che riproduca gli elementi cardine di un'immagine in prospettiva. Questo codice potrà essere utilizzato anche per la realizzazione di modelli tattili da fotografia e, naturalmente, per i singoli fotogrammi di un video. Per favorire la comprensione dei rapporti che legano uno spazio tridimensionale con la corrispondente immagine in prospettiva, abbiamo realizzato un modello tridimensionale cubico (concavo) in scala l:20 di una stanza con lato pari a $\mathrm{m} 4$ e pareti rimovibili, in cui sono presenti quattro solidi elementari (convessi): un cilindro, un cono, una sfera e una piramide (fig. I).

A partire da questo modello, abbiamo individuato 12 condizioni proiettive differenti (fig. 2), utili a evidenziare i principali parametri che determinano l'immagine prospettica, variando reciprocamente la posizione dell'oggetto rispetto al quadro, l'altezza del punto di vista rispetto al geometrale e la distanza del punto di vista dal quadro. Di ciascuna immagine è stato stampato un bassorilievo (fig. 3) utilizzando 5 curve di livello equidistanti mm I, in modo da simulare il corrispondente schema prospettico. La preventiva esplorazione tattile del modello tridimensionale potrà fornire al cieco la comprensione spaziale dello spazio concavo e degli elementi convessi presenti all'interno.

Successivamente, la lettura attraverso il tatto delle prospettive in bassorilievo permetterà di comprendere alcuni effetti tipici di una prospettiva/fotografia di cui i principali sono:

- lo scorcio (gli oggetti vicini appaiono più grandi di quelli lontani);

- l'interposizione dei volumi (gli oggetti posti davanti nascondono quelli collocati dietro);
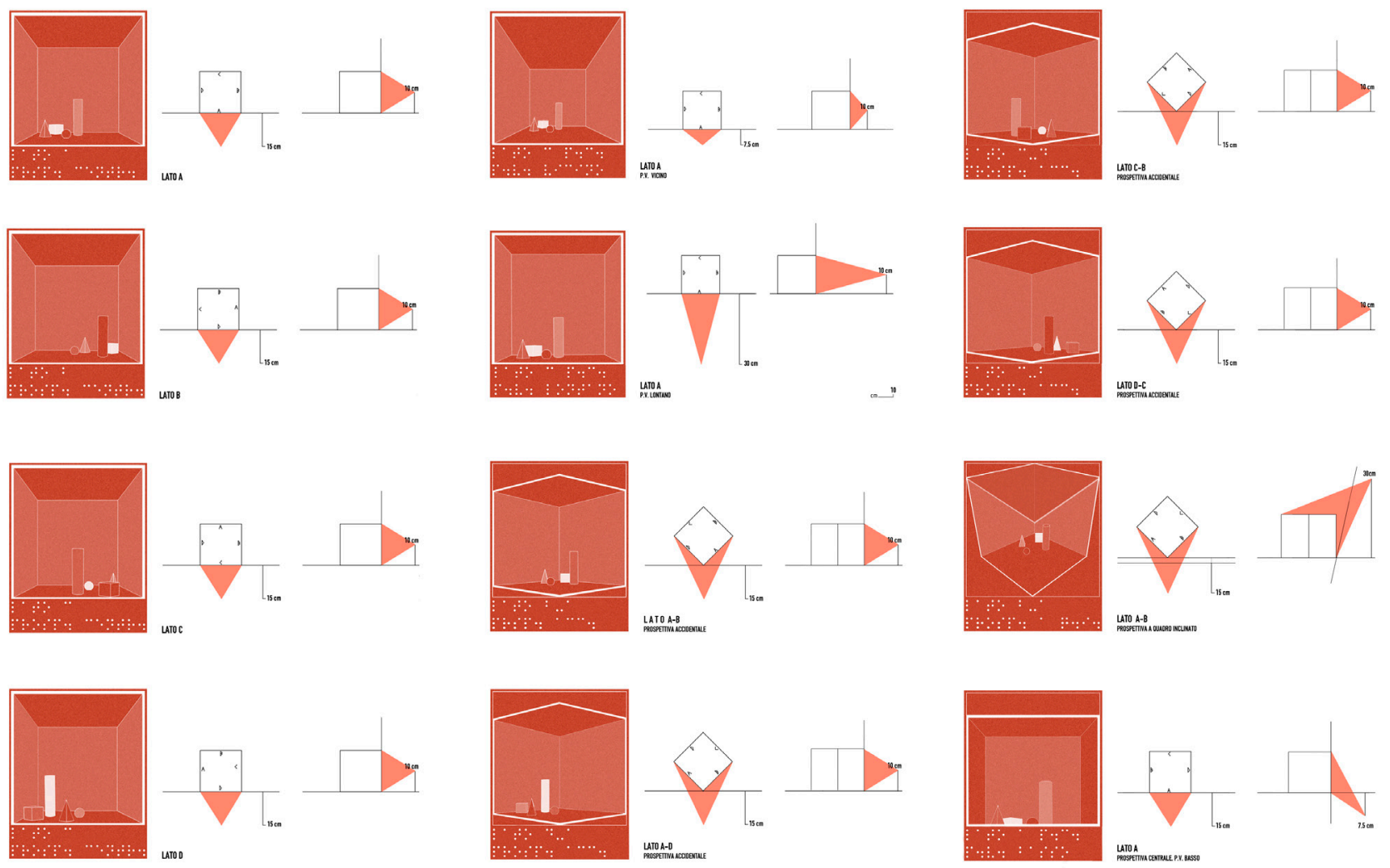

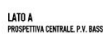


- gli effetti conseguenti alla variazione dell'altezza del punto di vista rispetto al geometrale (se alzo la linea di orizzonte 'domino' la scena; se la abbasso, gli oggetti appaiono più imponenti);

- gli effetti conseguenti alla lunghezza focale delle ottiche impiegate (ossia la distanza del punto di vista dal quadro): se mi avvicino, avrò un maggiore scorcio (effetto grandangolo) e la prospettiva sarà più accentuata, se mi allontano lo scorcio sarà minore (effetto teleobiettivo) e la prospettiva sarà più dolce e meno drammatica.

Al termine di questa fase, che in questa sede è stata presentata in estrema sintesi, avremo definito un codice 'minimo' per la comunicazione dello spazio tridimensionale (consapevolezza che il cieco possiede già) tramite un supporto tattile bidimensionale (bassorilievo) che costituisce un analogo dello spazio stesso.

\section{II film. Analisi visuale e sperimentazione su La finestra sul cortile}

La fase successiva del lavoro è consistita nella definizione delle qualità 'visuali' che caratterizzano la sequenza delle immagini che compongono un film, trascurando quindi tutte le informazioni relative alla diegesi, alla caratterizzazione dei personaggi, ai dialoghi, agli effetti sonori, ecc. Qualità che un cieco non può percepire e che non possono essere rese efficacemente con una descrizione verbale. Abbiamo suddiviso tutte le qualità visuali in tre categorie, riferite sia a singoli fotogrammi che a sequenze di fotogrammi (porzioni di ripresa) [6]:

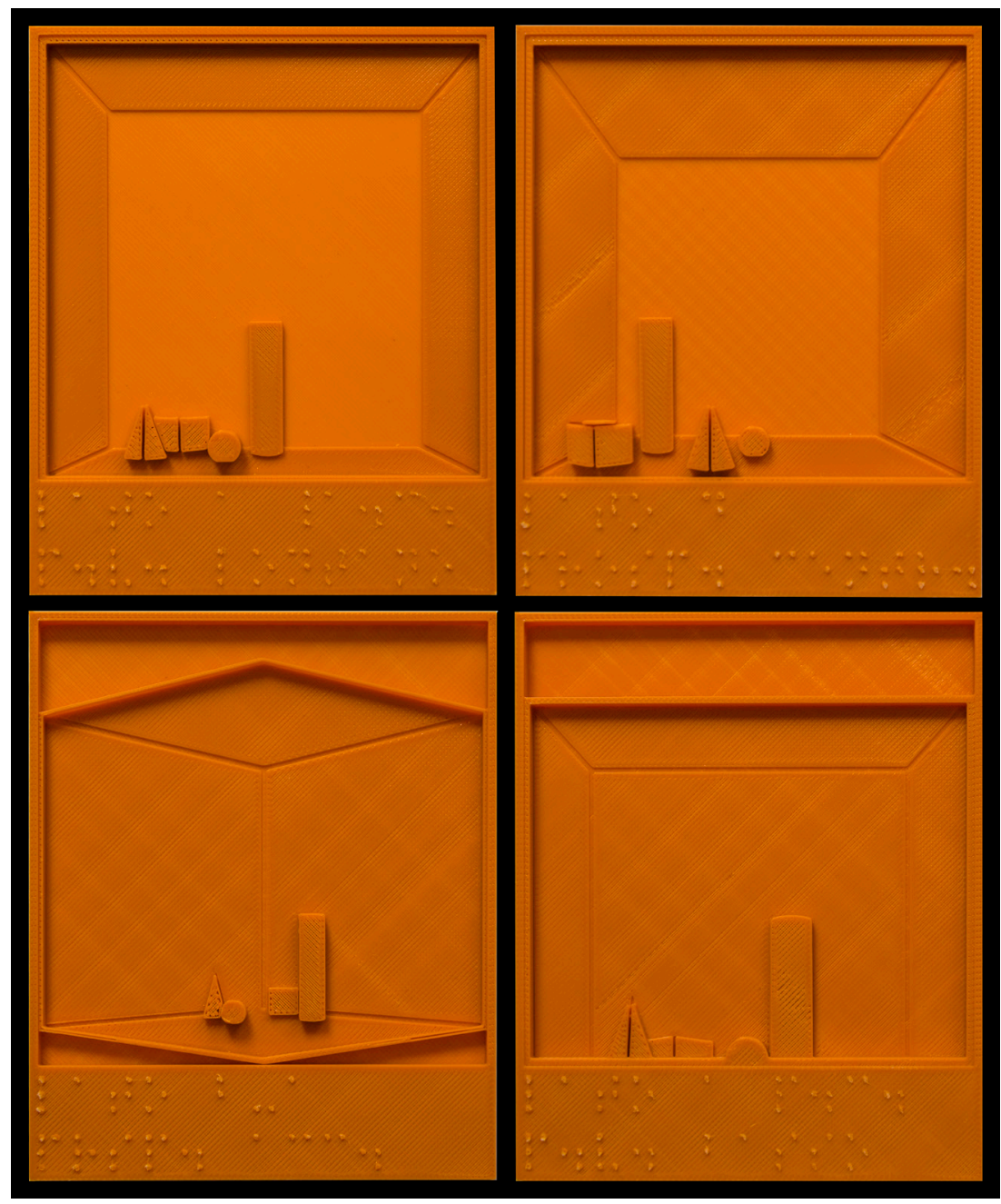


- il set (fig. 4), relativa agli elementi che caratterizzano lo spazio fisico della scena (luci, arredi, costumi ecc.);

- le riprese (fig. 5), comprendente le scelte legate alla cattura delle immagini: il tipo di pellicola utilizzata, l'inquadratura, i movimenti della macchina da presa, i cambi di ottica ecc.;

- il montaggio (fig. 6), riferita alle operazioni eseguite in fase di postproduzione (dissolvenze, cut-in e cut-away, sovraimpressione, controcampo ecc.).

Le qualità visuali complessivamente individuate sono 57 e sono state ulteriormente suddivise in tre gruppi:

- qualità che possono essere rese con un supporto tattile statico (bassorilievo): ad esempio, 'primo piano';

- qualità che possono essere rese solo con un supporto tattile dinamico (tablet a punti motorizzati): per esempio, 'dissolvenza';

- qualità che non possono essere rese con un supporto tattile statico né con uno dinamico: ad esempio, 'overlapping'.

Questa suddivisione è funzionale non solo alla realizzazione dei supporti in rilievo, ma anche per valutare preventivamente l'efficacia della traduzione di un'immagine su supporto tattile: ovviamente, un film di azione in esterni, dinamico dal punto di vista delle riprese e dell'editing sarà molto complesso (o addirittura impossibile) da realizzare, a differenza di un film con inquadrature statiche e sequenze di durata elevata, ripreso prevalentemente in interni.

La fase successiva consiste nellindividuazione dei fotogrammi e delle scene chiave da trasformare in supporto tattile. II loro numero è variabile e dipende da diversi parametri, di cui i principali sono il tipo di supporto tramite cui realizzare i bassorilievi (statico o dinamico) e le caratteristiche del film. Nella sperimentazione effettuata su tre lungometraggi (Rear Window di Alfred Hitchcock, Metropolis di Fritz Lang e The Draughtsman's Contract di Peter

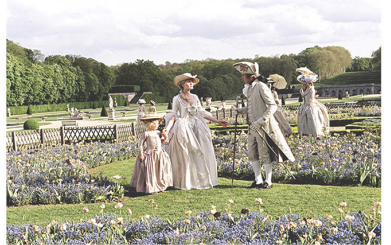

fotogrammi in cui gli ambienti natural rivestono un ruolo caratter

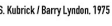

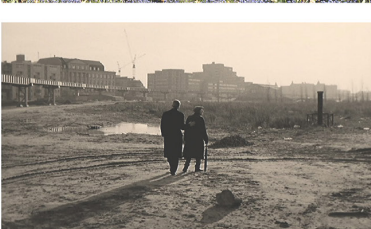

1 AMBEENT NATURA

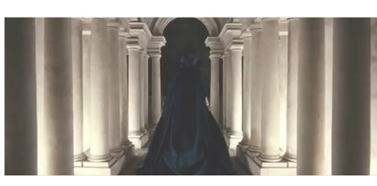

102

Totogrammi in cui la sce
un ruolo caratterizzant

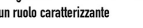

сІт⿺尢丶

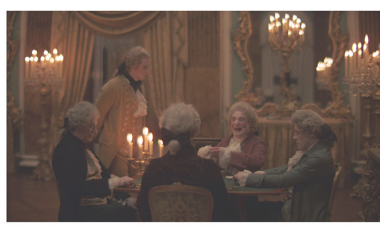

fotogrammi in cuil larchitettura riveste u

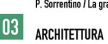
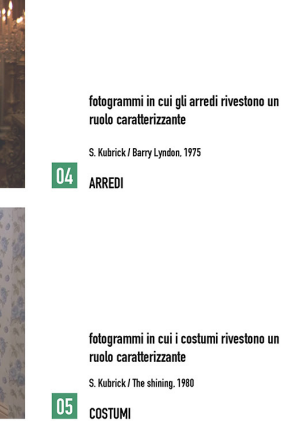
4 ARREDI
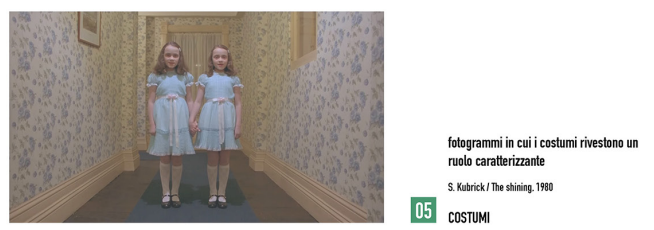

CosTUMI S. Kubrick/ Barry Lymoon. 1975
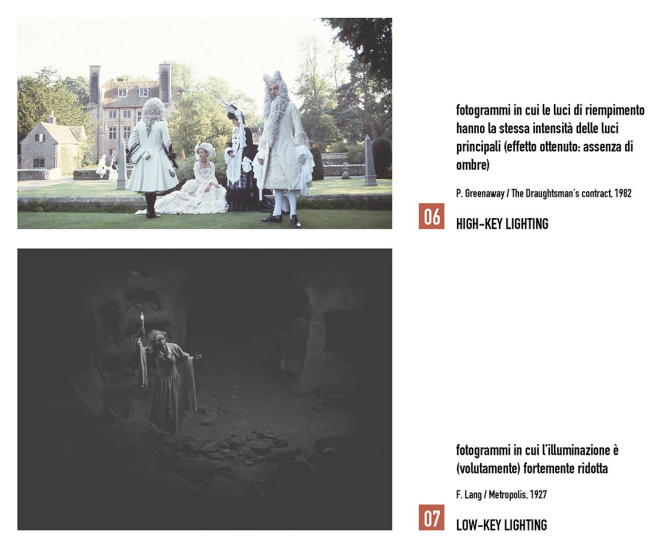

06 HIGH-KEY LIGHTING

fotogrammi in cuil ililuminazione

F. Lang Meropolas. 1927

07 LOW-KEY LGHTING
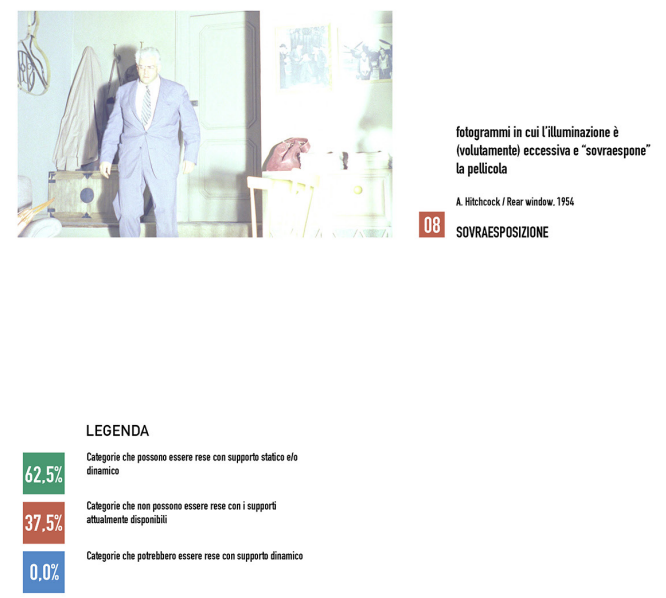

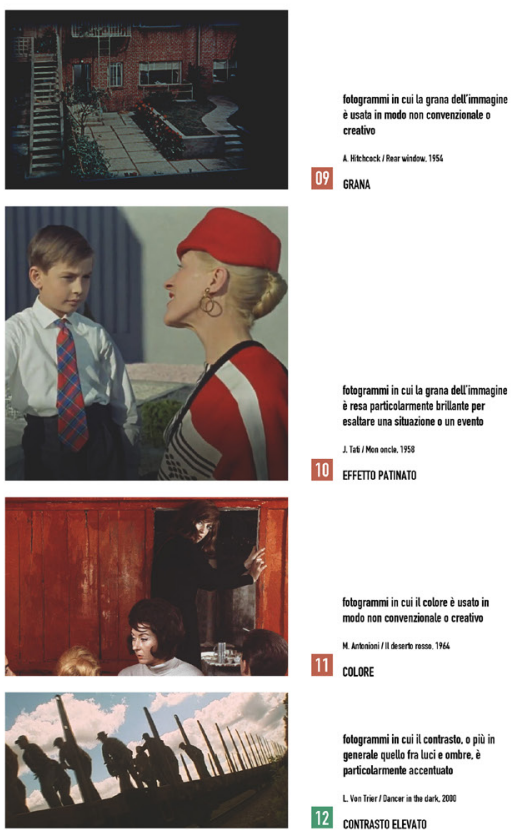

11 CODPE
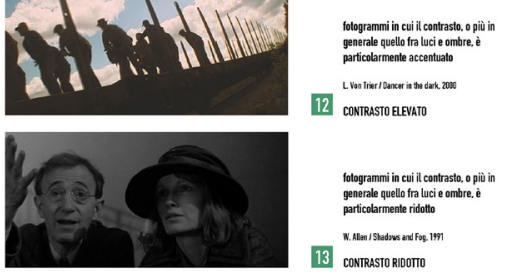

12 contrasto Ger
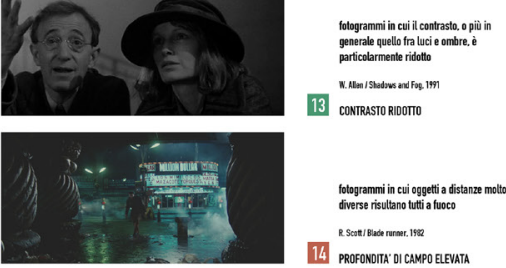

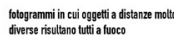

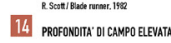

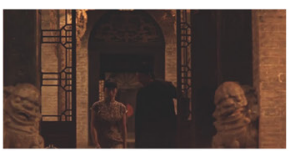

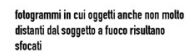

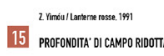
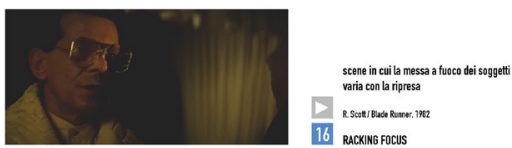

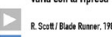
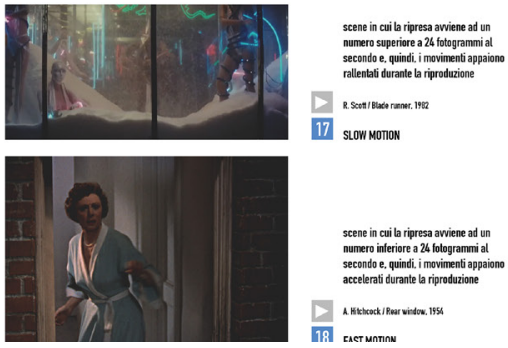

17 slow Moті1

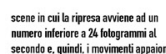

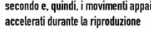

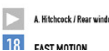
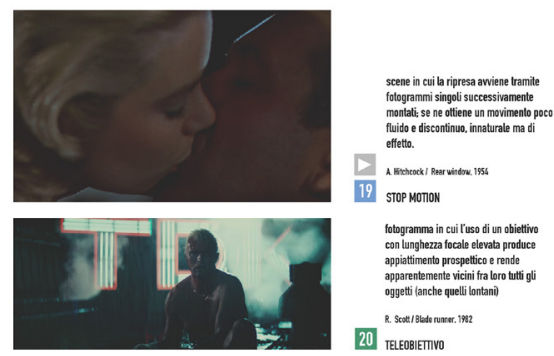

19 STrop MOTOK

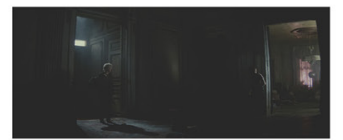

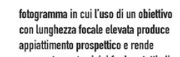

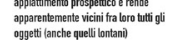

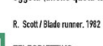

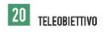

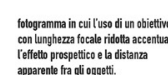

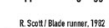

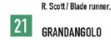

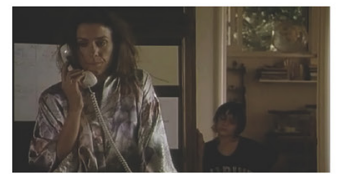

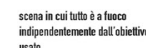

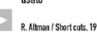

22 PAMFocius
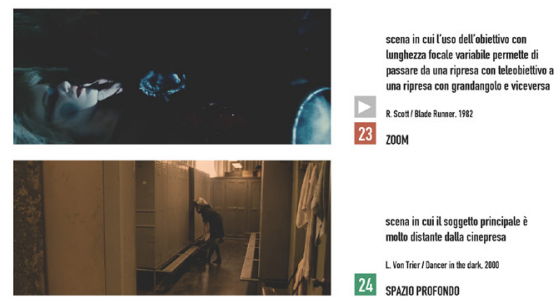

232000

Scena in cuili soggetlo principale

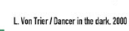

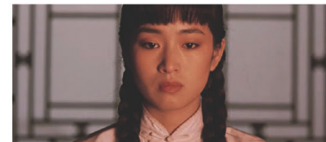

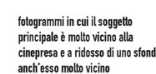

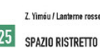
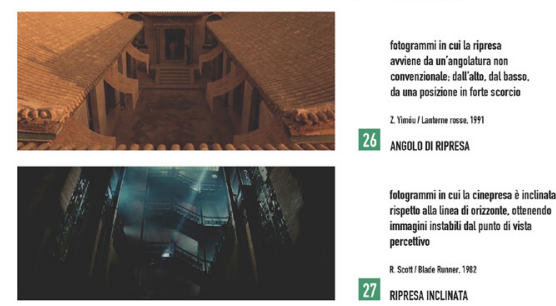

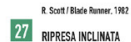

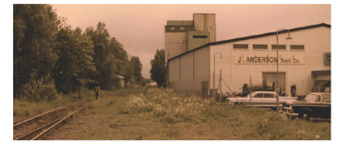

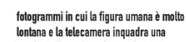

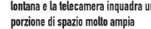

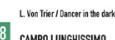

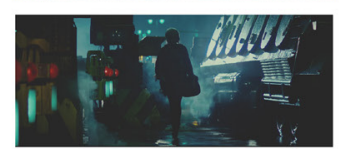

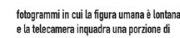

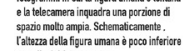

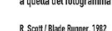

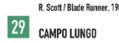

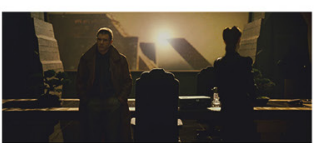

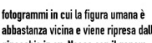

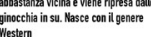

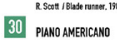

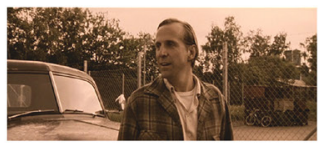

Totogrammini in cuil lifigura unana è vicína

inte tax tano

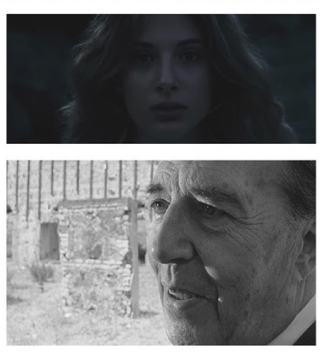

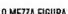

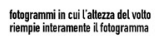

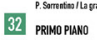

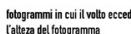

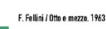

33 Primo pulvo siretro

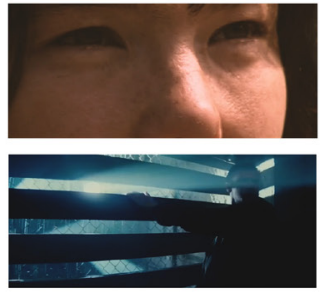

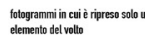

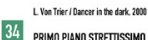

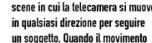
un soggatto. Quando il moviment
del soggetlo e minimo si deffrise
Retraning

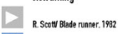
35 RPPESA MOElLE

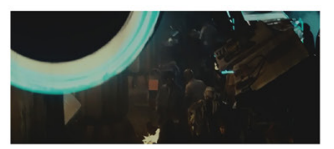

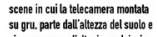

simumeve
direzione

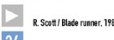

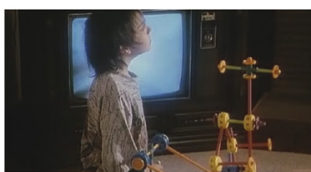

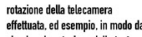

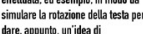

"panorarices"

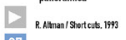

37 PA

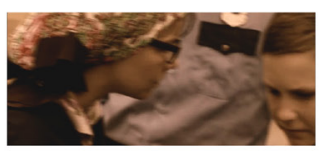

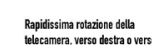

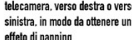

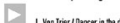

38 WHIP PaN

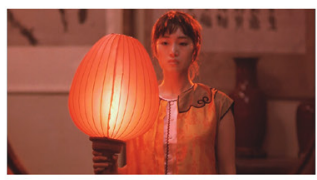

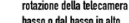

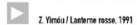

39 TLI

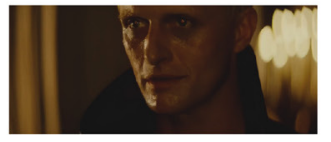

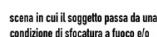

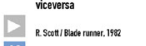

4609725050202817

Fig. 5. Categorie visuali:

le riprese. 

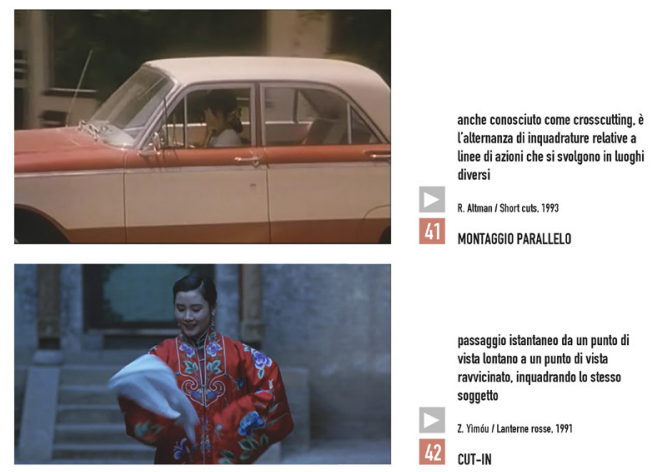

41 montagGio parallelo

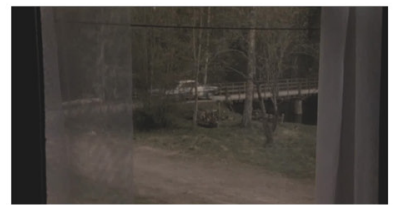

è linverso del cut-in 43 L Von Trier I Dancere in the dark. 2000
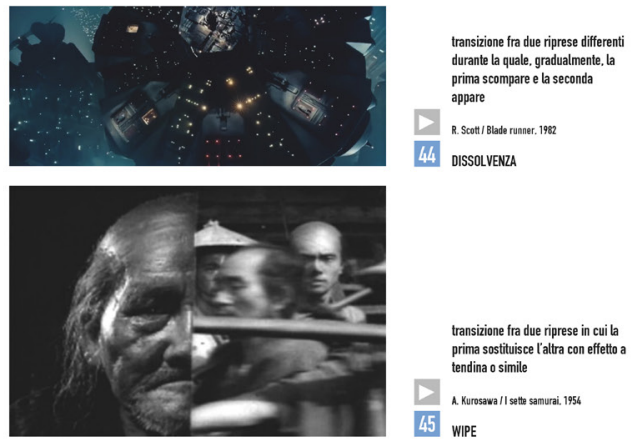

44 R. Sotssollelateruma rawicinalb
soggetto
son

42 CUT-IN

transizine fra due riprese in cuile prima sositiusis
tendina o simile

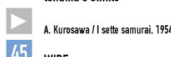

45 WIPE

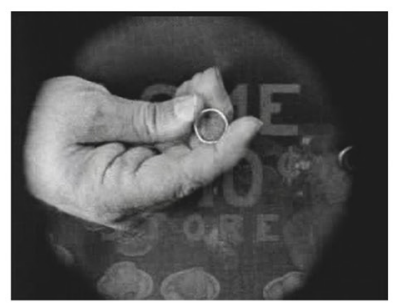

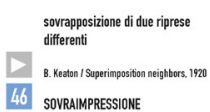
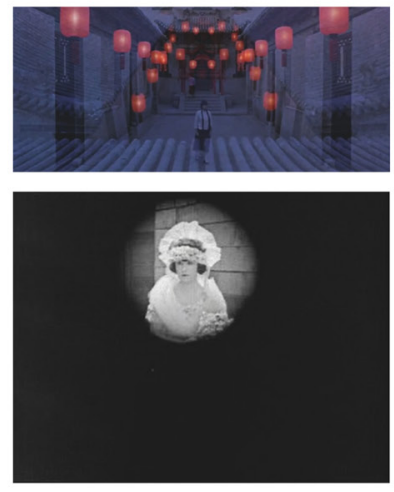

maschera circolare che siallarga (iris-in) o si restringe (iris-outt su
scena $D_{\text {B. Keash }}^{\text {scena }}$

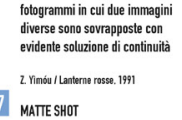

48 IRIS

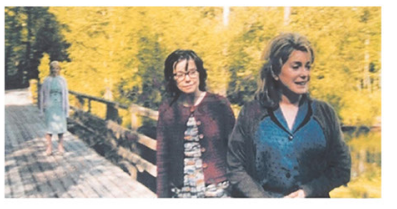

taglio della parte centrale di una ripresa
in modo da collegare bruscamente la parte iniziale e quella finale. Gli oggetti psivione allation

L. Von Thier I Dancer is the dark. 2001 49 JUMP-CUT

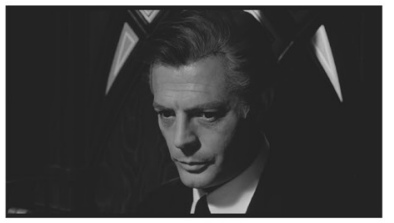

scene in cuil ta telecamera si posiziona dal soggetto inuuarrato recedentememe
F. Fellinil totoe merzo. 1960

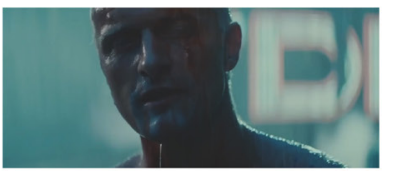

50 RIPRESA SOGGETIIVA

scene che riprendono un soggetto
dal punto otivista opposto rispettoa dal punto divista opposto
quello della precedente

inguadratura

R. R. Scot/8bataf Rumer, 1982

51 conrRocaMpo

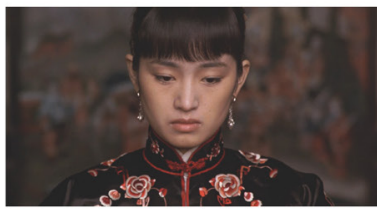

scene che riprendono oli occhidi iun
soggetto e immediatamente dopo ció soggetto e immediatmente do
che il soggetto sta guardando

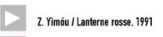

52 ErelNEMAтCH

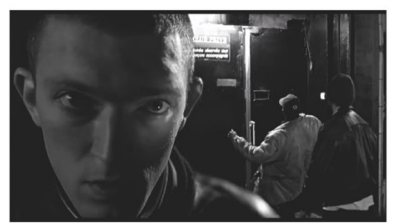

sourapposisione di due inquadrature diverse ma simili dal punto divista
puramente grafico o metaforico

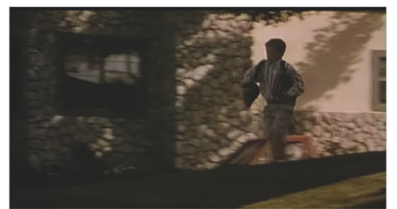

53 GRAPHICMATCH

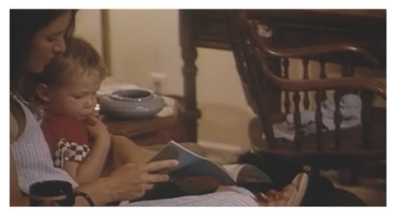
interdanu un soggetto in movimerts

R. Retrana / Start cuts. 1999

54 MarCH ON ACTION

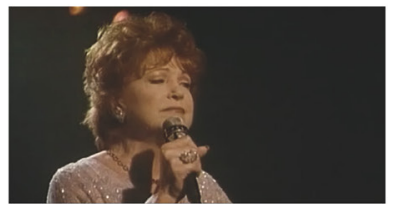

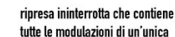
tutele le motulazioni ditun unica
scena. senzar ricorrere al montaggi R. A. Atrana/ / Shortctut.1993

55 PIAvo SEauerza

ripetizione di un'azione inquadrata do R. Altman / Stortctuts. 1995 56 OVVRLAPPING

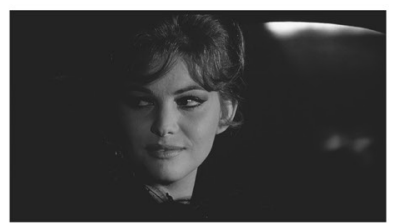

scene in cuile inprese dei personagg

C.

57. RERPOPOIEIIONE

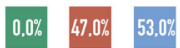


Greenaway), abbiamo potuto analizzare e catalogare le difficoltà conseguenti alle riprese in esterni, all'uso di costumi e arredi sontuosi, allintensità della luce, ai movimenti di macchina e agli effetti che si possono ottenere col solo montaggio analogico. Si tratta di una fase dello studio che in questa sede, per brevità, si omette.

Nel caso specifico di RearWindow abbiamo individuato 30 fotogrammi/riprese chiave (fig. 7), che ovviamente non possono rendere tutta la complessa visualità del film ma che comunque possono fornire un supporto utile a integrare la comprensione degli eventi e riprodurre alcune scelte del regista strettamente legate alla visualità.

Una volta individuati i fotogrammi chiave, abbiamo realizzato i modelli 3D degli spazi oggetto delle riprese. In generale, la costruzione del modello 3D può awvenire in modi diversi (fig. 8):

- ricostruzione da elaborati grafici, se esistono i progetti esecutivi del set;

- rilievo in situ, se le riprese sono state effettuate in spazi pubblici accessibili;

- restituzione prospettica da fotogramma singolo, qualora ci siano elementi di dimensioni certe nelle inquadrature;

- ricostruzione da stereo coppie, nel caso in cui il film sia stato girato con camera tereoscopica. Nel caso di Rear Window, la costruzione è awvenuta tramite restituzione prospettica da fotogramma singolo ed è stata favorita dal fatto che le riprese dell'intero lungometraggio sono state effettuate esclusivamente all'interno di una stanza e sul cortile del caseggiato su cui la stessa affaccia (fig. 9).

Abbiamo quindi realizzato due modelli analogici in acido polilattico (PLA) (fig. I0): uno relativo al cortile del caseggiato in cui si svolge l'intera vicenda (scala I: I00) e uno relativo alla
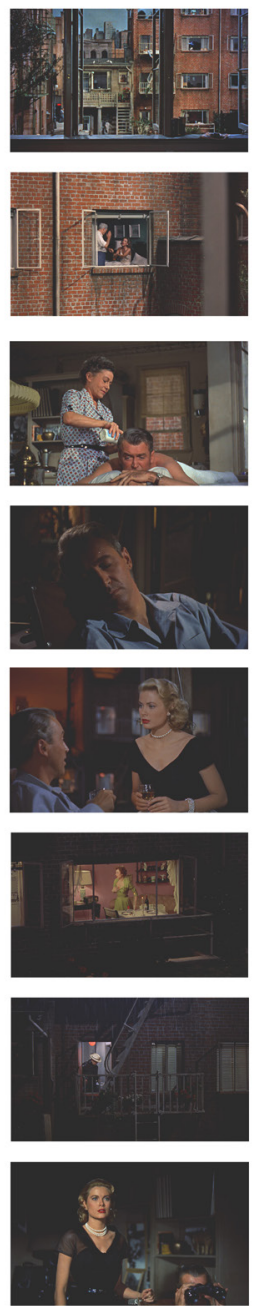
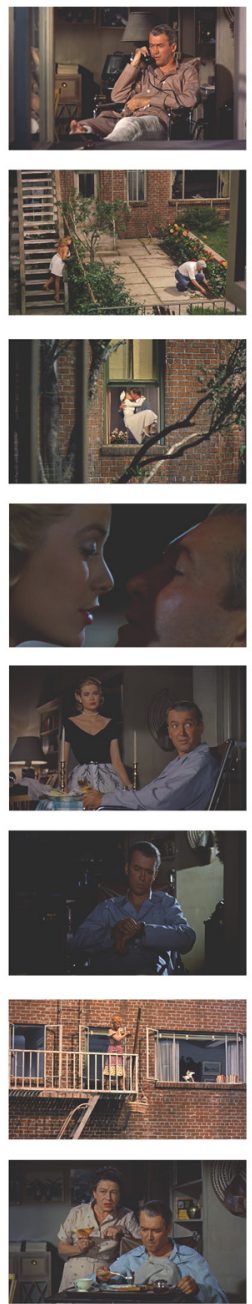
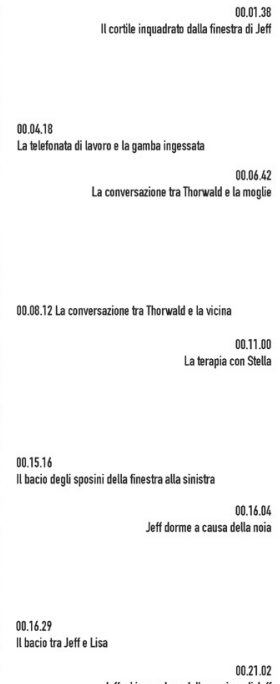

wons

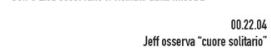

wases
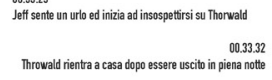

conss

10.36.58
Una conne ta scendere in cortle il cane con la cerrucola
\[ 00.49 .2 \]

Jeffe Lisa spiano Thorwald conil binoc.29.21
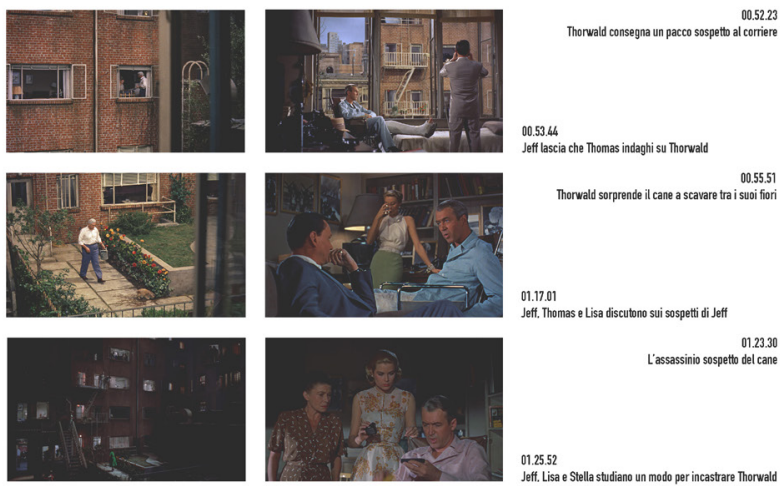

01.17 .91

Jeft. Thomas e Lisa discutono sui sospettit i i efff
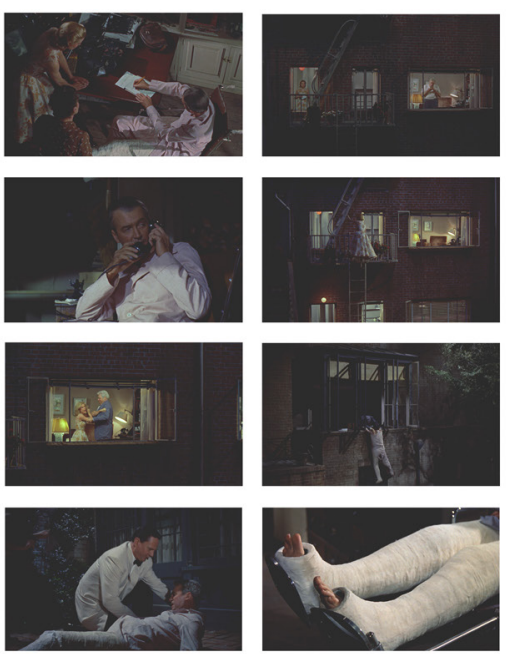

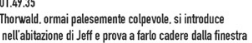

Thomas riesce a savare Jeff dolla caduta

01.5151
Jefts 5 iritova con ben due gambe ingessat. ma las sua vita

LA COMPOSIZIONE DEL FILM

00.51 .21
Jeff e Sitlela pootzanor la scena del crimine 
stanza da cui il protagonista osserva gli eventi (scala 1:20). Attraverso la preventiva esplorazione tattile dei due modelli tridimensionali, prima della visione del film, il cieco potrà avere un'idea delle caratteristiche dello spazio in cui si svolgeranno gli eventi.

Successivamente sono stati realizzati, sempre in PLA, il bassorilievo dei fotogrammi chiave che possono essere resi tramite supporto statico; il criterio è stato quello di sintetizzare la scena tramite 5 layer sovrapposti, ognuno dei quali è compreso in una superficie piana sporgente I mm rispetto a quella sottostante (fig. I I). II bassorilievo comprende una didascalia in Braille con indicazioni utili a collocare la scena all'interno dello sviluppo della storia (fig. 12).

\section{Conclusioni}

I risultati della ricerca sintetizzata in questo studio permettono di effettuare il lavoro preparatorio per la costruzione dei bassorilievi utili a descrivere i fotogrammi e le scene chiave di un film statico e privo di effetti speciali come Rear Window. Nel caso specifico, abbiamo scelto 30 fotogrammi che successivamente abbiamo tradotto in bassorilievi. Un'operazione del genere ha dei limiti che la costringono a rimanere a livello sperimentale: fra questi, la mole di bassorilievi da realizzare, il costo di modellazione e prototipazione, la necessità di un supporto audio integrativo per consentire al cieco di scegliere il bassorilievo corrispondente alla scena nel momento esatto, l'impossibilità di riprodurre alcune qualità legate alle riprese (come i movimenti di macchina) e tutte le qualità relative al montaggio. Molti di questi limiti saranno superati appena sarà disponibile il tablet a microbolle motorizzate; con questo dispositivo, l'intera sequenza di immagini di un film potrà essere tradotta in rilievi che si succedono fluidamente e in tempo reale durante la proiezione. Ma anche in questo caso, occorre fare un'eccezione per alcune qualità (legate ai cromatismi, all'uso delle luci, alle sfocature e al mosso intenzionale, ecc.) per le quali il surrogato dei bassorilievi non è adatto ed è necessario effettuare dei test direttamente sul dispositivo motorizzato. Analoghe difficoltà

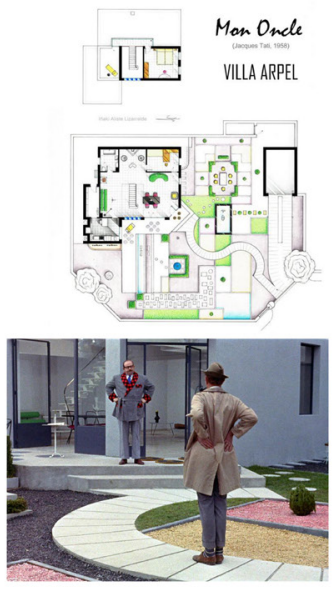

Viene effetutuat tramite itridisegno degli elaborati

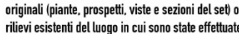
rileviesist
riprese

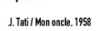

01 RICOSTRUZIONE DA Elaboran GRafCI

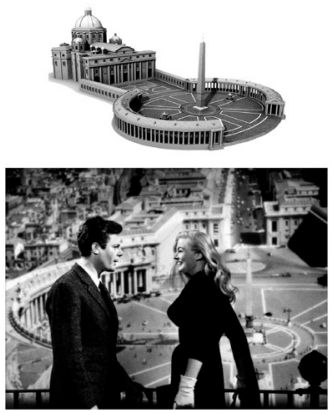

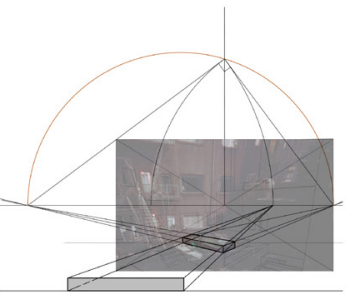

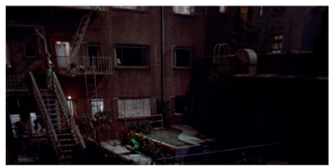

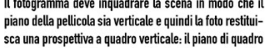

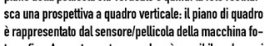

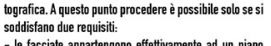

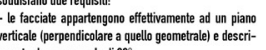
vono tra laro un angolod di 90

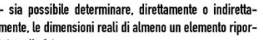

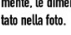

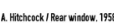

03 RESTTUUIINE PROSPETTICA DA FOTOGRAMMA SINGOLO
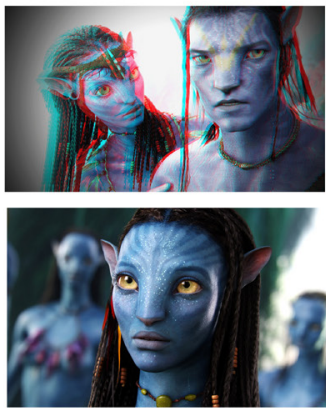

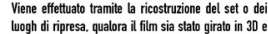
quindi siano disponibiil gi steteregrammi a anaglifi..

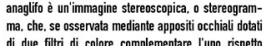

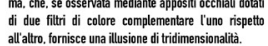

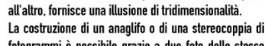

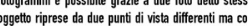

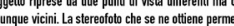
di determinare le misure degli oggetti ipresesi J. Cameren/hatara: 2009

04 RICOSTRUZLONE DA STEREOCOPPIE 
Fig. 9. Restituzione grafica della stanza e del caseggiato in cui è girato Rear
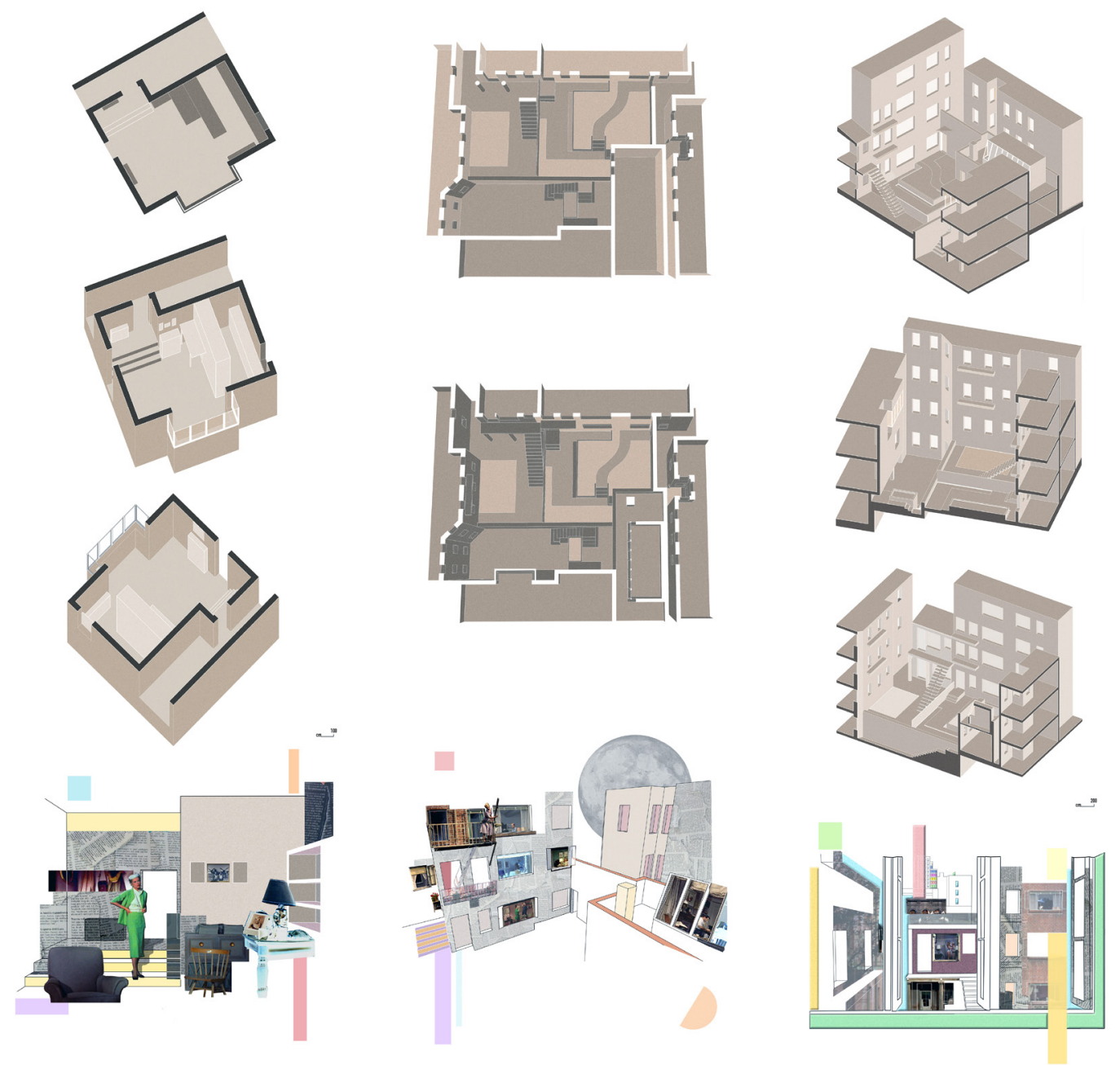

Fig. 10.Viste del modello tridimensionale del case giato in cui è ambientato Rear Window e della stanza di Jeff.

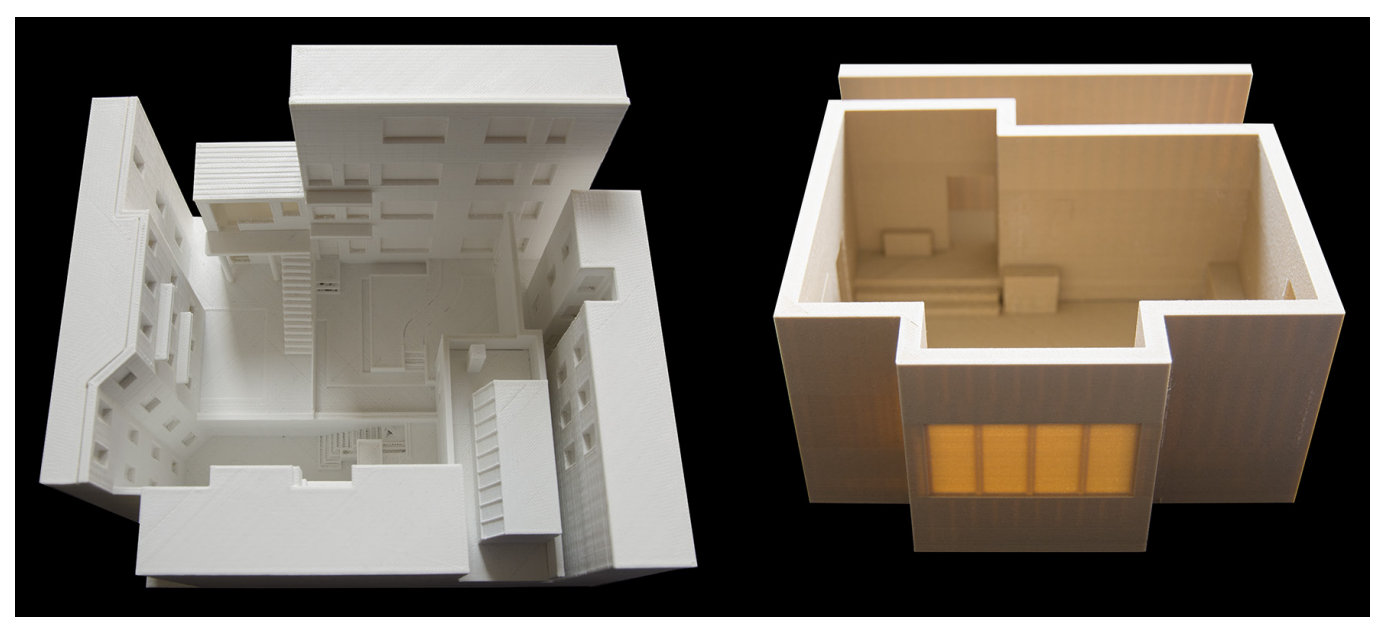




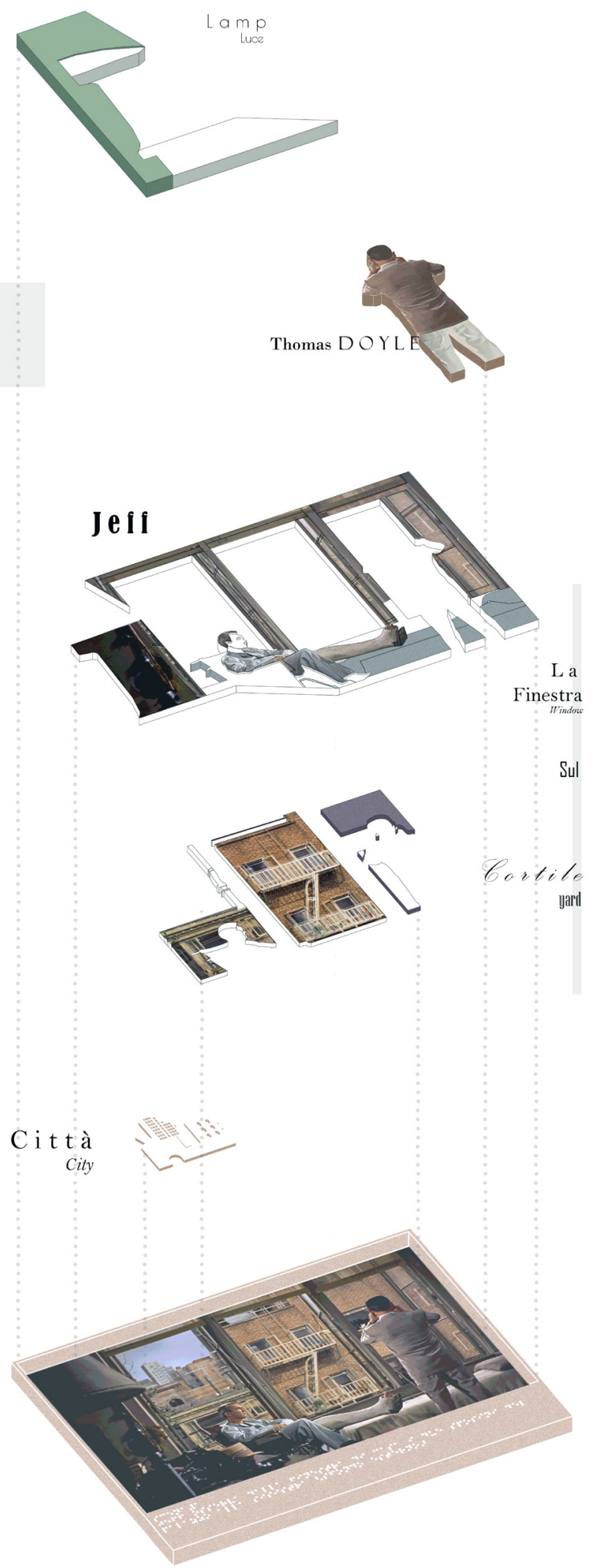


si pongono per effetti di ripresa o di montaggio che si sviluppano in tempi molto ridotti, come ad esempio il whip pan. II limite principale, tuttavia, è legato all'indisponibilità di un supporto che presumibilmente sarà commercializzato a breve e potrà offrire un ulteriore sviluppo alla ricerca in corso [7].
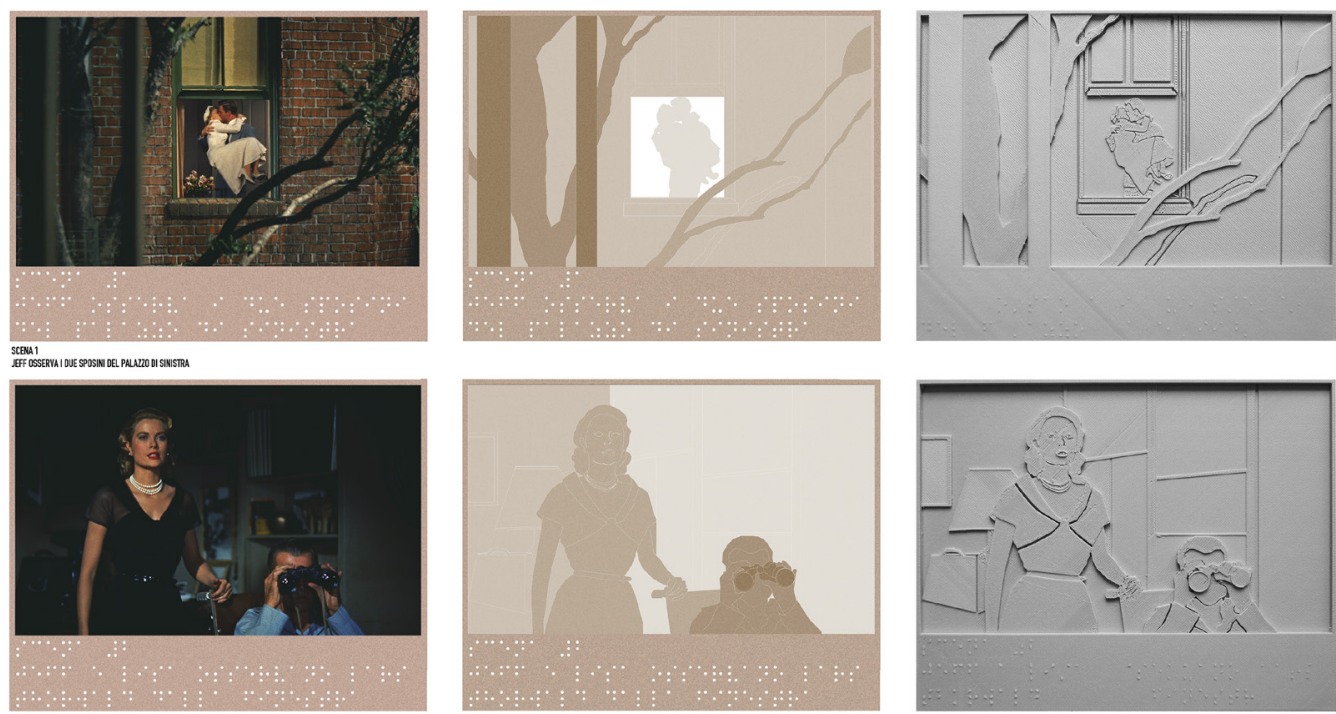

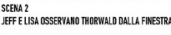

Fig. 12. Dal fotogramma alla suddivisione in layer a bassorilievo.

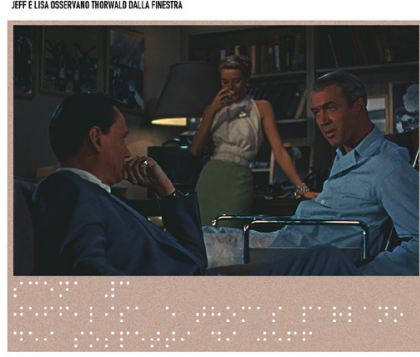

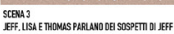
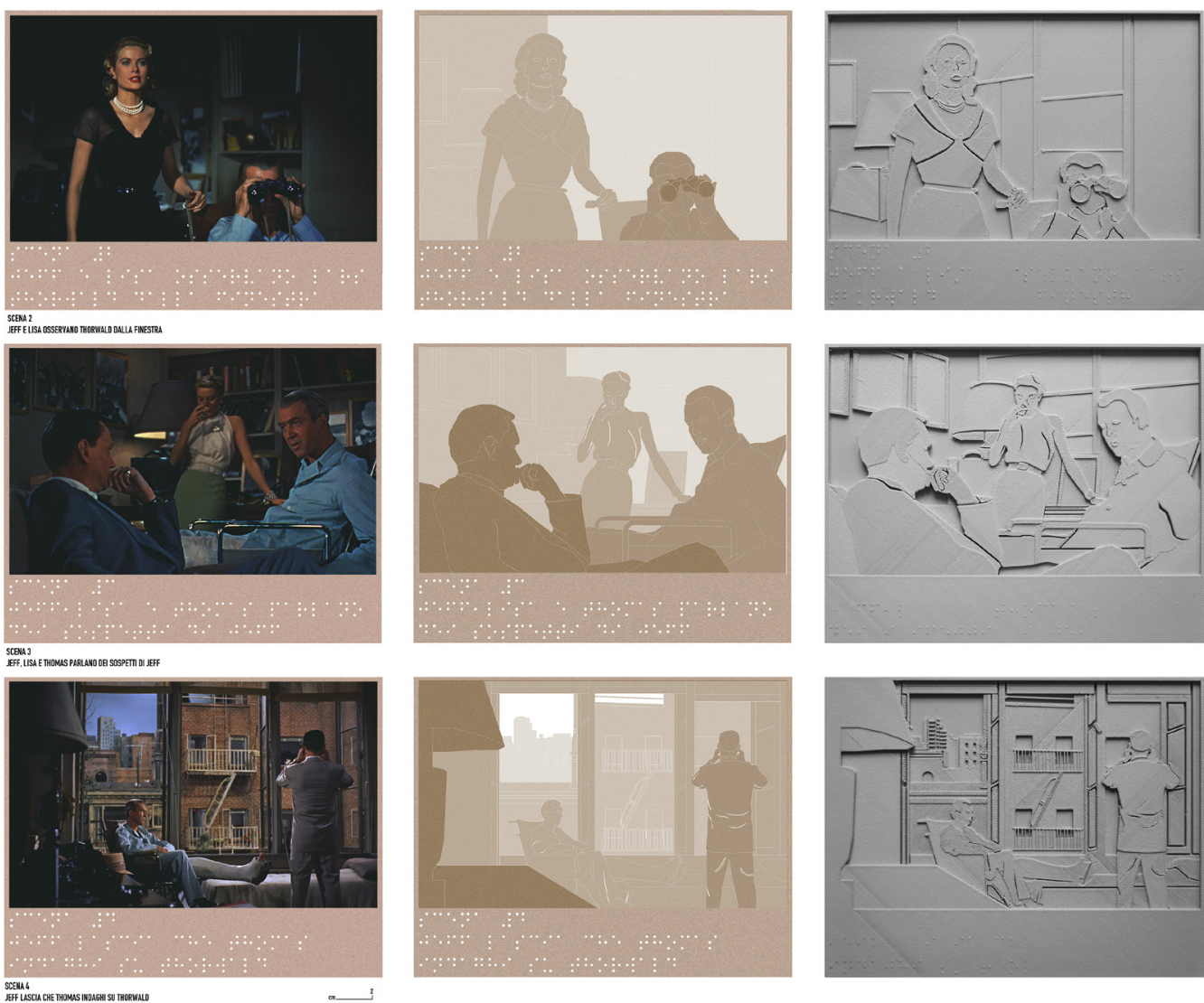

\section{Note}

[I] Sull'argomento si veda: Brambring 2006.

[2] I supporti di audiodescrizione disponibili possono essere suddivisi in due categorie: le cineteche audio online (come cineaudioteca.it o moviesfortheblind.com) e le app per dispositivi mobili (come Moviereading o Greta). Si tratta in entrambi i casi di supporti che aiutano la comprensione di ciò che viene proiettato sullo schermo attraverso una descrizione verbale che, in tempo reale, si sovrappone ai dialoghi e ai suoni della pellicola originale.

[3] Su questo argomento, la bibliografia e le esperienze effettuate sono innumerevoli e in questa sede non è possibile ripercorrerle tutte. Un riferimento valido sulla grafica, soprattutto dal punto di vista pedagogico, è il lavoro di Edman: Edman 1992. Molto interessanti sono anche le sperimentazioni in cui i sono i non vedenti a realizzare le riprese fotografiche; le esperienze più innovative sono consultabili su <3dphotoworks.com> e su <blindwithcamera.org>

[4] I dispositivi elettromeccanici adatti a riprodurre la scrittura Braille esistono da diversi decenni, ma hanno avuto una evoluzione 
pressoché nulla in quanto la riproduzione di un testo attraverso una griglia di punti costituisce un'operazione molto semplice. Lo stesso sistema (griglia di punzoni metallici o in plastica sollevabili meccanicamente) è stato utilizzato anche per riprodurre forme rudimentali, ed esistono diversi prodotti sul mercato (per i testi: <blitab.com/> per le forme elementari: < feelif.com/ blindpad. eu/> o il recentissimo Tactile Pro <powerct.kr/>). II limite di tutti questi dispositivi è costituito dal fatto che la griglia è comunque vincolata a un interasse fra i punti che non può scendere al di un certo valore e quindi le forme riproducibili sono molto schematiche. Da alcuni mesi è stata annunciata, da diversi centri di ricerca, l'imminente prototipazione un nuovo tablet basato non più su punzoni ma su decine di migliaia di punti motorizzati (microbolle poste sotto una guaina elastica), in grado quindi di restituire forme molto più complesse e articolate, e soprattutto il movimento delle forme stesse; al momento però il prodotto non è stato ancora presentato.

[5] Sulla questione della prospettiva occorre ricordare la posizione di Erwin Panofsky e quella ad essa contrapposta di Decio Gioseffi: Panofsky 1927; Gioseffi 1957.

[6] II lavoro prende spunto da un'esperienza, integralmente rielaborata, sviluppata presso l'University of Yale: <filmanalysis.coursepress.yale.edu>. Per gli aspetti relativi al film in generale, si veda: Rondolino 20 I 8; per il montaggio si è fatto riferimento a: Cassani 2013.

[7] Daniele Colistra ha definito la struttura della ricerca e ha scritto il testo; Giada Puccinelli ha realizzato le sperimentazioni sui modelli tattili, l'analisi dei film e le immagini.

\section{Riferimenti bibliografici}

Brambring Michael (a cura di). (2006). Early Intervention with Infants and Preschoolers who are Blind. Würzburg: Bentheim.

Cassani Diego (20 I3). Manuale del montaggio. Tecnica dell'Editing nella comunicazione cinematografica e audiovisiva. Novara: UTET Università.

Edman Polly K. (1992). Tactile Graphics. New York: AFB Press.

EmplerTommaso, Fusinetti Alexandra (2019). Rappresentazione visuo-tattile. Comunicazione tattile per i disabili visivi. In Belardi Paolo (a cura di). Riflessioni. L'arte del disegnolil disegno dell'arte. Atti del $41^{\circ}$ Convegno Internazionale dei Docenti delle Discipline della Rappresentazione, Perugia 19-2 I settembre 2019. Roma: Gangemi, pp. I563-1572

Gioseffi Decio (1957). Perspectiva artificialis. Per la storia della prospettiva. Spigolature e appunti. Trieste: Università degli Studi di Trieste.

Grassini Aldo (20 16). Per un'estetica della tattilità. Ma esistono dawero le arti visive? Roma: Armando Editore.

Grassini Aldo, Sòcrati Andrea,Trasatti Annalisa (20 I 8). L'arte contemporanea e la scoperta dei valori della tattilità. Roma:Armando Editore.

Levi Fabio, Rolli Rocco (1994). Disegnare per le mani. Manuale di disegno in rilievo. Torino: Zamorani.

Munari Bruno (1986). I laboratori tattili. Bologna: Zanichelli.

Panofsky Erwin (1927). Die Perspektive als "Symbolische Form". Leipzig-Berlin:Teubner. (Trad. it. La prospettiva come "forma simbolica" e altri scritti (196I). Milano: Feltrinelli.

Rondolino Gianni,Tomasi Dario (20 I 8). Manuale del film. Racconto, linguaggio, analisi. Novara: UTET Università.

Sacchetti Anna Maria (2005). Vedere con le mani. Marina di Massa: Edizioni Clandestine.

\section{Autori}

Daniele Colistra, Università degli Studi Mediterranea di Reggio Calabria, daniele.colistra@unirc.it Giada Puccinelli, Università degli Studi Mediterranea di Reggio Calabria, giadapuccinelli@libero.it

Per citare questo capitolo: Colistra Daniele, Puccinelli Giada (2020). Cinema per i non vedenti. Dispositivi tattili per la fruizione/Cinema for the blind Tactile devices for enjoyment. In Arena A., Arena M., Brandolino R.G., Colistra D., Ginex G., Mediati D., Nucifora S., Raffa P. (a cura di). Connettere. Un disegno per annodare e tessere. Atti del $42^{\circ}$ Convegno Internazionale dei Docenti delle Discipline della Rappresentazione/Connecting. Drawing for weaving relationships. Proceedings of the 42th International Conference of Representation Disciplines Teachers. Milano: FrancoAngeli, pp. 31 29-3154. 


\title{
Cinema for the Blind. Tactile Devices for Enjoyment
}

\author{
Daniele Colistra \\ Giada Puccinelli
}

Abstract

This paper presents the first results of a research on the topic of tactile devices for the fruition of films by blind people. This topic has been rarely addressed so far, and its principles have not been systematized yet. Therefore, it offers many possibilities for experimentation. Research is still ongoing and has been simultaneously developed for the tactile perception of photography and two-dimensional graphic arts. This paper will focus on cinematography, while referring to principles which are mostly valid for other figurative forms.

The auxiliary tools currently available to a blind person to enjoy a film consist exclusively of audio supports which integrate the dialogues between the characters and offer minimal information relating to visual qualities. The audio integrations, based on the verbal description, are not suitable for describing many expressive components related to visuality (shots, optics, video camera movements, choices related to film editing etc.) which, as is evident, constitute essential elements to fully appreciate a movie.

The most advanced studies and the first prototypes made in the digital tactile devices industries (motorized microspheres tablets), even if not yet available on a commercial scale, could expand an economically relevant sector of great scientific interest for design science. In addition, it would allow inclusiveness and greater access to culture to a category of disadvantaged people. In this paper we describe the method developed to achieve the bas-reliefs of the key scenes of a film, applying it to Alfred Hitchcock's Rear Window and trying to focus on the weaknesses and potentials of this experience.

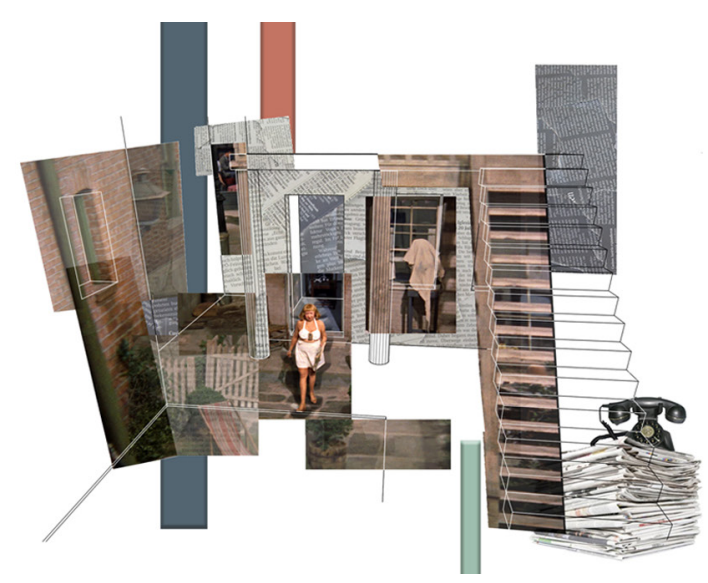

3142

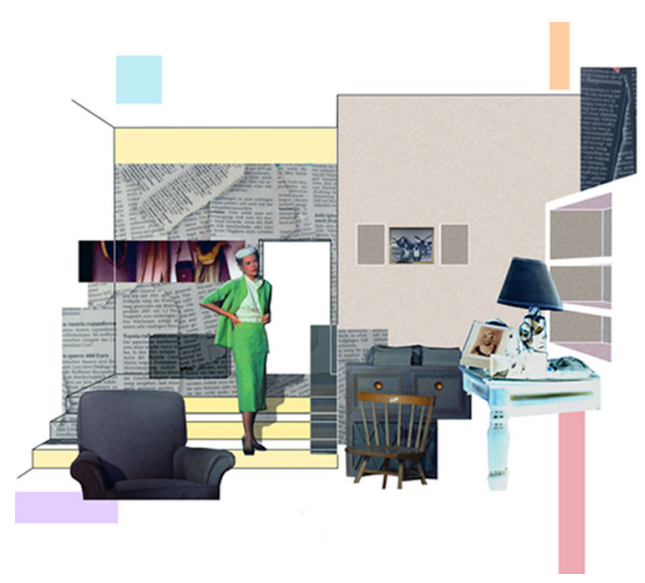

doi.org/| 0.3280/oa-548.17| 


\section{Context and state of the art}

A blind person has a perfect spatial awareness of an environment he knows; he can move at ease in space and manages to relate to it with his own body. Touching the objects, the blind acquires analytically many information which, however, during the haptic phase, is separated from a unitary three-dimensional reference scheme. Through sight we perceive convex objects (for example: a ceramic vase) and concave spaces (for example: the interior of a church) first in a general and synthetic way; then, in some cases, also analytically. For a visually impaired person, the procedure is reversed: the objects and spaces are reconstructed by adding up the information acquired, by parts, through touch or movement within them.Verbal communication, on the other hand, is transmitted through a code (Braille) which reproduces all the phonetic signs within writing and, therefore, the transmission of information occurs without any loss of meaning. For this reason, the method of communication with and among blind people occur mainly with verbal, written or sonorous forms, avoiding as much as possible the use of spatial information, colour or any element related to visibility [I].

The tools currently available for promoting the enjoyment of a film are also based on supplementary audio supports [2]: a narrative voice briefly describes the scene, the gestures and the actions, in some cases the movements of the camera or the choices related to the editing; however, numerous aesthetic intentions, mainly visual, which are the basis of cinematography and which should be communicated, albeit partially, remain excluded. Through audio integrations, the blind person will be able to better understand the film but will not be able to appreciate many of its intrinsic qualities, precisely those related to visuality.

Why, unlike the media for photography and painting [3], hasn't there been a similar development in the film industry? Mainly for two reasons: first of all because a blind person is still able to 'understand' the film even only through hearing, but also because the numerous images that make up a feature film require the creation of a large number of bas-reliefs which, however, would be suitable only for reproducing static scenes, free of camera movements and quick actions. The recent announcements relating to the mass production of an interactive relief tablet, suitable not only for braille writing but also for the perception of complex shapes, could quickly overcome the current and apparently insurmountable difficulties [4].

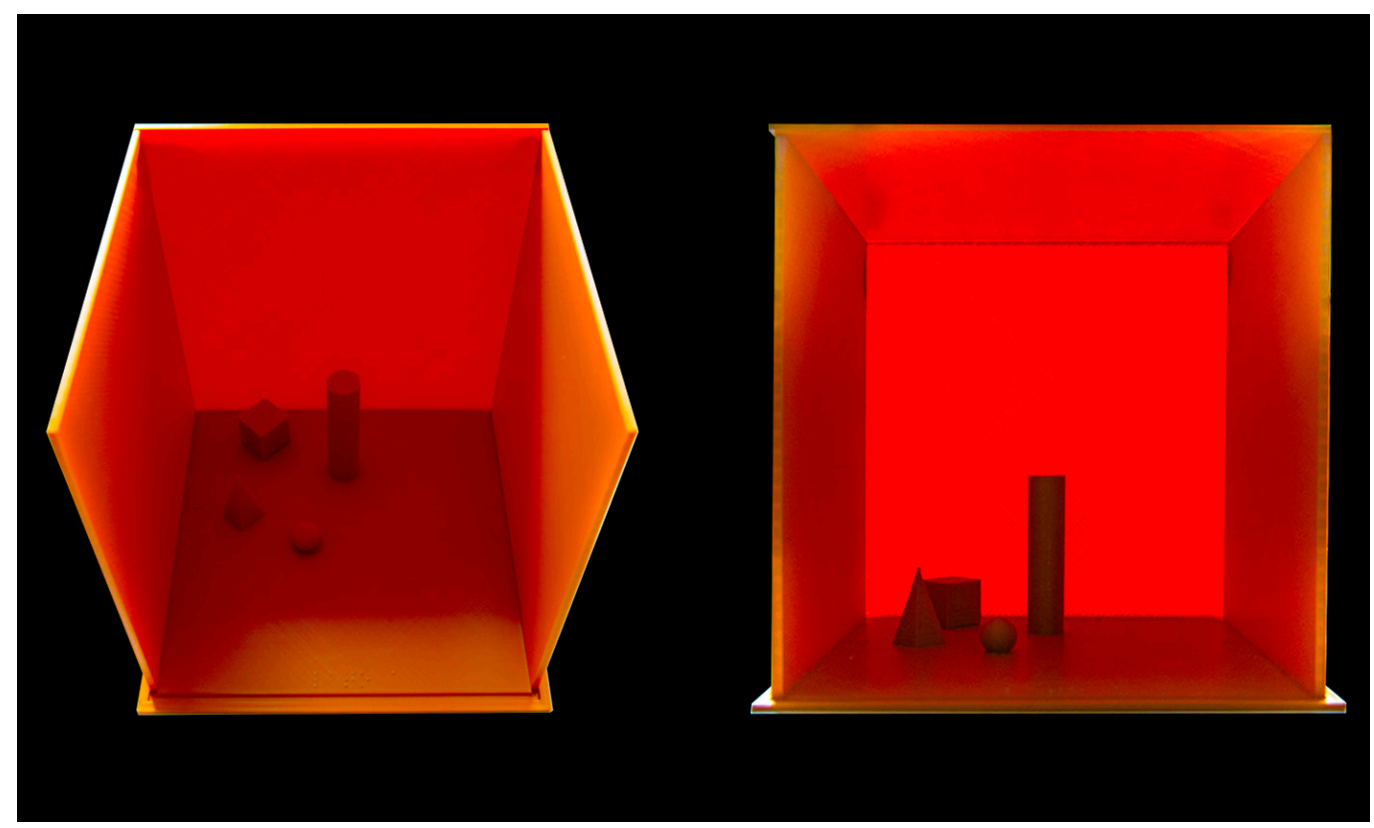




\section{Aptic and proprioception. An experimental model for spatial perception}

The first problem we faced is related to the unitary representation of a concave space. People see space through images that are formed on the retina, and the perspective is the most analogous form of the representation to what we see with the eyes, despite being equipped with elements of conventionality, symbolicity [5] and, therefore, needs reading keys for its correct interpretation. We tried to define an elementary code for the communication of three-dimensional space through a tactile support (bas-relief) that reproduces the key elements of a perspective. This code can also be used for the creation of tactile photography models and, of course, for the individual frames of a video. To understand the relationships that link a three-dimensional space with the corresponding perspective image, we have created a cubic (concave) three-dimensional model of a room, in 1:20 scale, with a $4 \mathrm{~m}$ side and removable walls. On the floor there are four elementary solids (convex volumes): a cylinder, a cone, a sphere and a pyramid (fig. I). Starting from this model, we have identified 12 different projective conditions (fig. 2), useful for highlighting the main parameters that determine the perspective image, by mutually changing the position of the object relative to the vertical projection plane, the height of the point of view relative to the horizontal plane and the distance of the point of view. We printed a bas-relief of each perspective image (fig. 3) using 5 equidistant I mm level curves, in order to simulate the corresponding perspective scheme. The preliminary tactile exploration of the three-dimensional model guarantees the blind spatial understanding of the concave space and of the convex elements inside. Subsequently, the cognitive experience through the touch of the bas-relief perspectives will allow to understand some typical effects of a perspective / photograph. These are the main ones: - the foreshortening (neighbouring objects appear larger than distant ones);
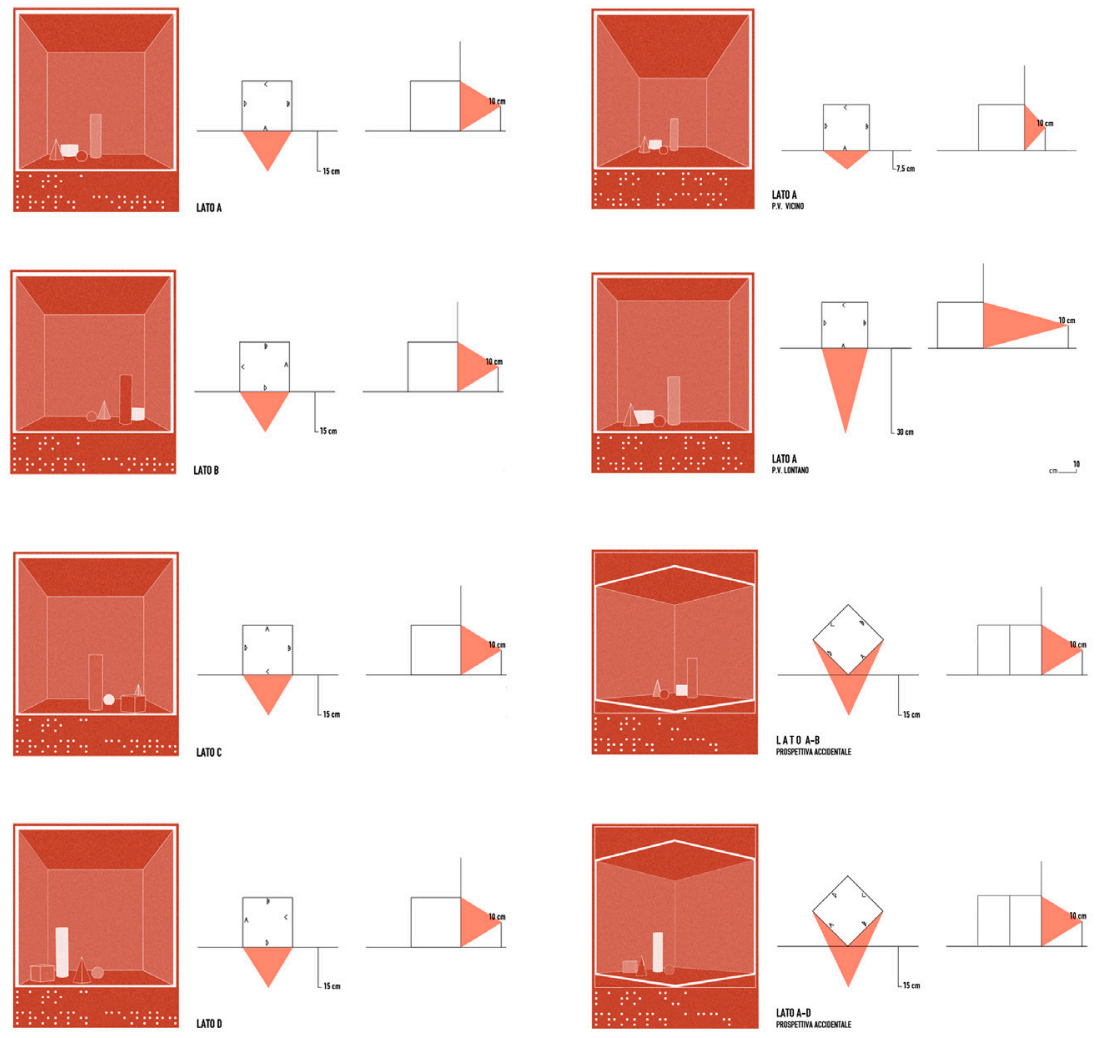
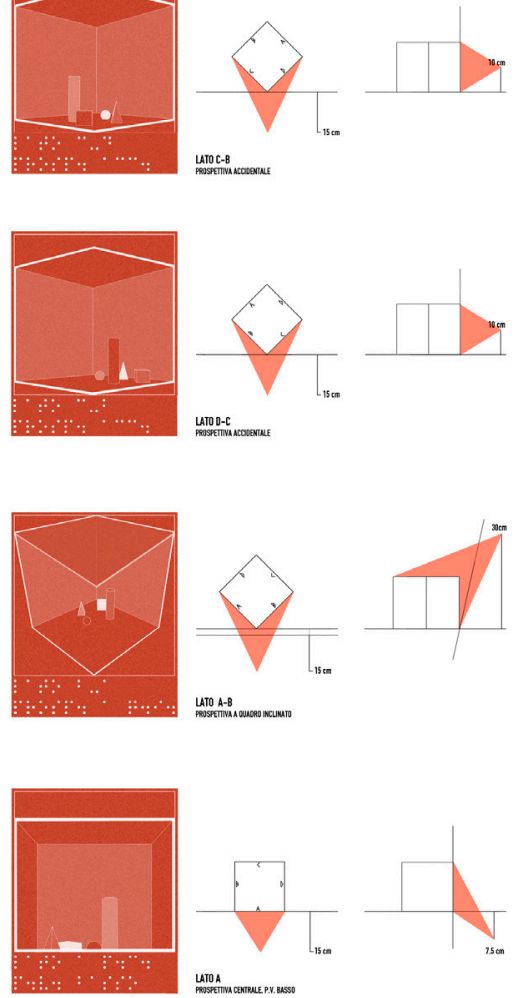

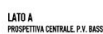


the horizontal plane (if the horizon line is raised, the observer 'dominates' the scene; conversely, if you lower it, the objects looks more impressive than in reality);

- the effects resulting from the focal length of the optics used (ie the distance of the point of view): if you reduce it, you will have a greater glimpse (wide angle effect) and the perspective will be more accentuated; if you increase it, the glimpse will be less (telephoto effect) and the perspective will be sweeter and less dramatic.

At the end of this phase we have therefore defined a 'minimum code' for the communication of three-dimensional space (awareness that the blind person already has) through a two-dimensional tactile support (bas-relief) which constitutes an analogue of the space itself.

\section{The film. Visual analysis and experimentations on Rear Window}

The next stage of the work consists in defining the 'visual' qualities that characterize the sequence of images that make up a film, thus neglecting all the information relating to diegesis, the psychology of the characters, dialogues, sound effects, etc. These are qualities that a blind person cannot perceive and which cannot be effectively rendered with a verbal description. We have divided all the visual qualities into three categories, referring both to single frames and to frame sequences (movie shooting portions) [6]:

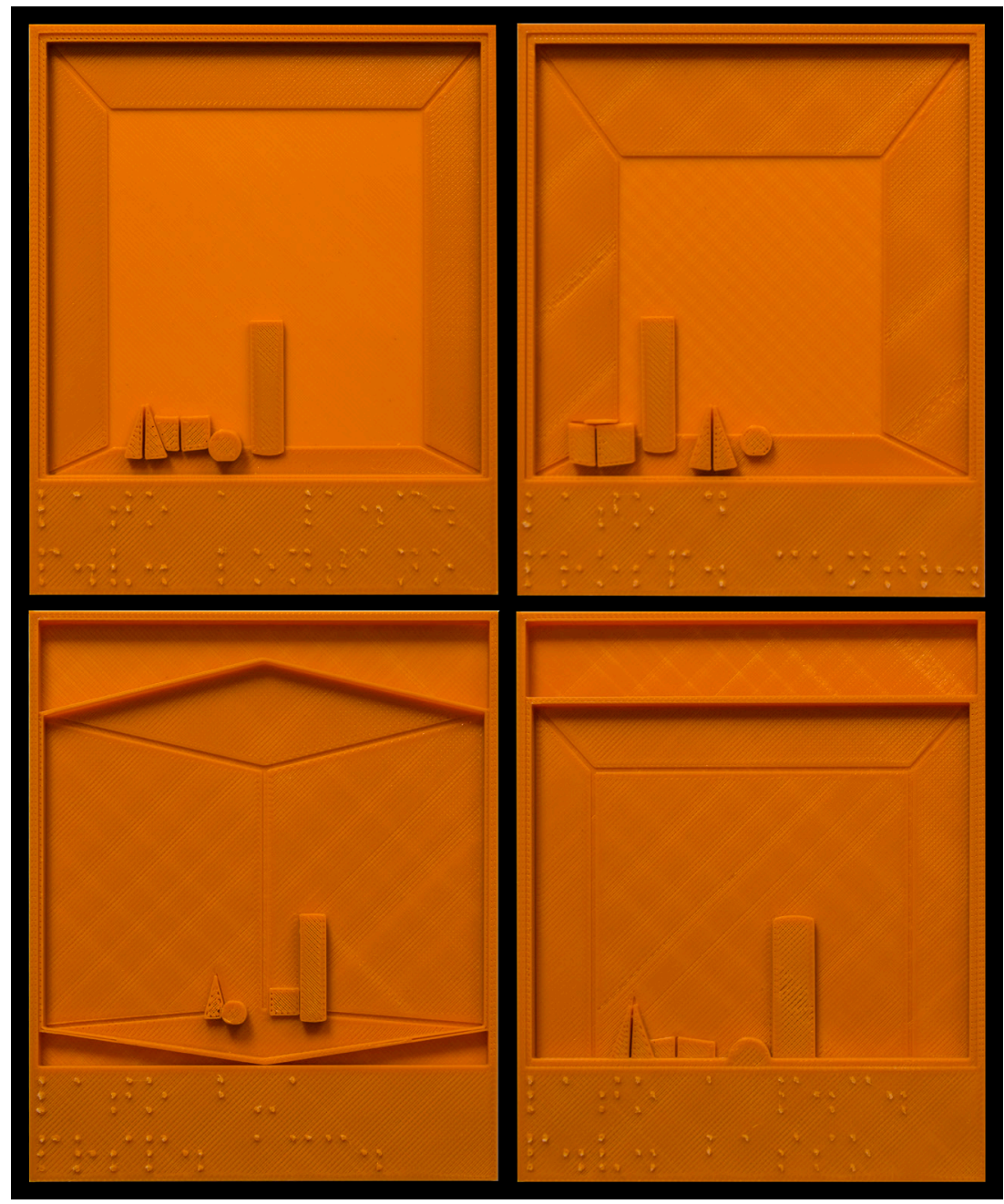


- staging (fig. 4), relative to the elements that characterize the physical space subject of the shot (lights, furnishings, costumes, landscapes, etc.);

- shooting (fig. 5), including the choices related to the capture of the images: the type of film used, the depth of field, the shot, the movements of the camera, the changes of optics etc.; - editing (fig. 6), referred to the operations performed in postproduction (fades, cut-in and cut-away, overlay, reverse shot etc.).

The visual qualities identified are 57 and have been further divided into three groups:

- qualities that can be described with a static tactile support (bas-relief): for example, 'close-up';

- qualities that can be rendered only with a dynamic tactile support (motorized point tablet): for example, 'fading';

- qualities that cannot be rendered with a static or even a dynamic tactile support: for example, 'overlapping'.

This division is functional not only for the realization of the bas-reliefs, but also for assessing in advance the effectiveness of the translation of the complex system of images of a film on a tactile support: obviously, an action film outdoors, dynamic from the point of view of filming and editing will be very complex (or even impossible) to be translated into a physical object, as opposed to a film with static shots and long duration sequences, mainly taken indoors.

In the experimentation carried out on three feature films (RearWindow by Alfred Hitchcock, Metropolis by Fritz Lang and The Draughtsman's Contract by Peter Greenaway), we analyzed and catalogued the difficulties resulting from shooting outdoors, the use of sumptuous costumes and furnishings, the variations of the intensity of lights, machine movements and the effects that can be obtained with analogical editing. This phase of the study, for brevity, has been omitted here.

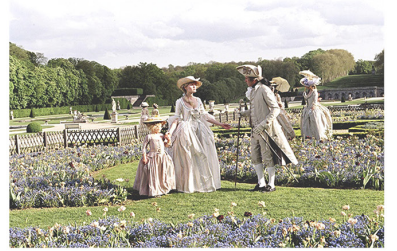

fotogrammi in cui gli ambienti natural rivestono un ruolo caratteri

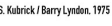

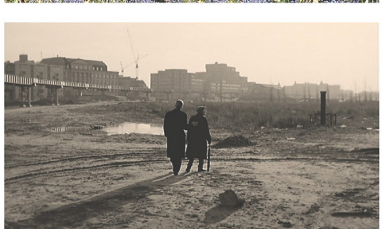

1 AMBEENT NATURAL

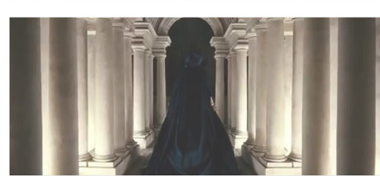

02

Totogrammi in cuil la scent
un ruolo caratterizante

СІTஷ̀

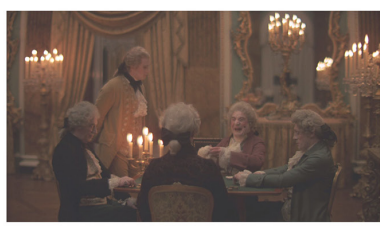

fotogrammi in cuil 'architettura riveste 4

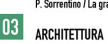

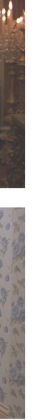

fologrammi in cui gli arredi ivestono un

ARREDI
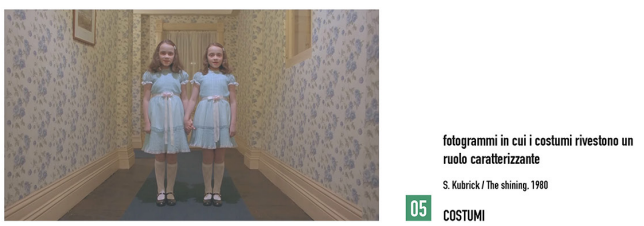
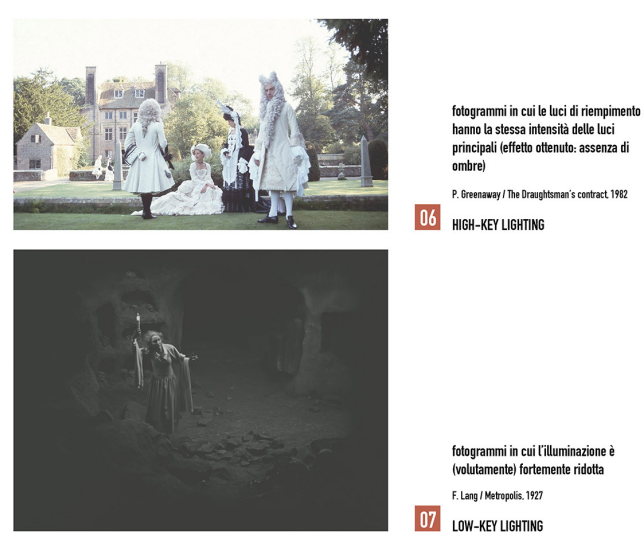

06 HIGH-KEY LIGHTING

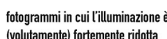

F. L. ang Metetopals. 1927

07 LOW-KEY LGHTING
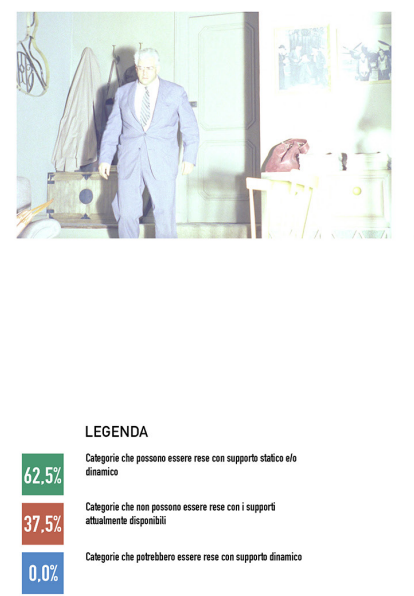

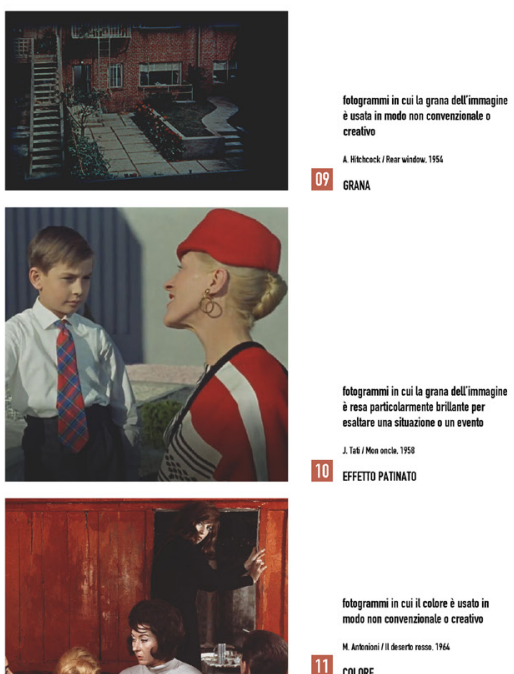

10 eFFetropatiNaI
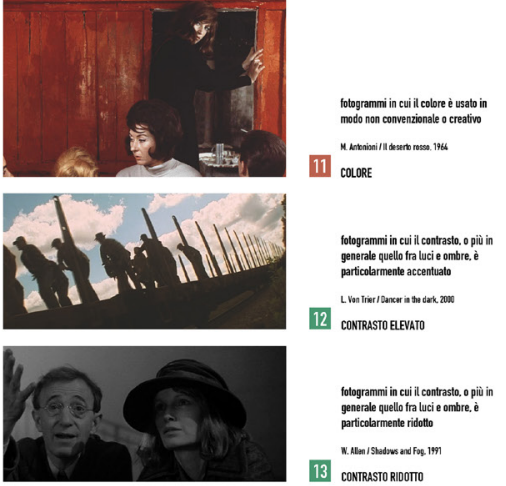

12 contrasto Ger
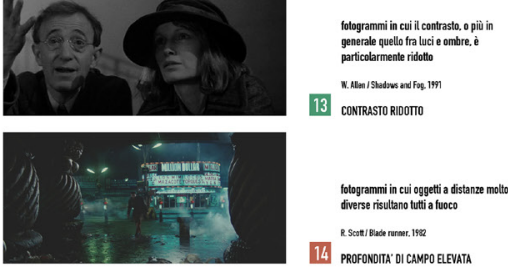

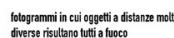

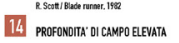

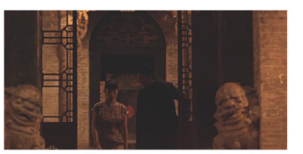

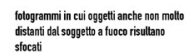

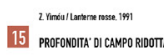
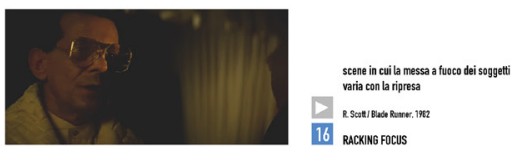

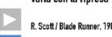
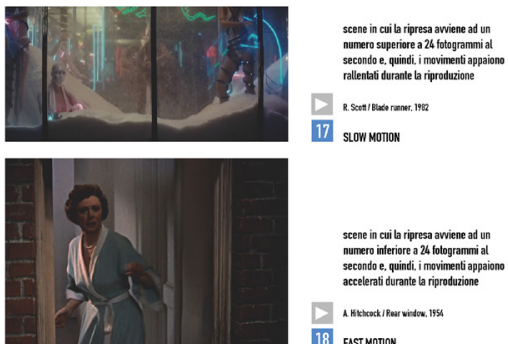

17 slow Moті1

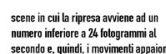

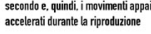

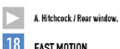
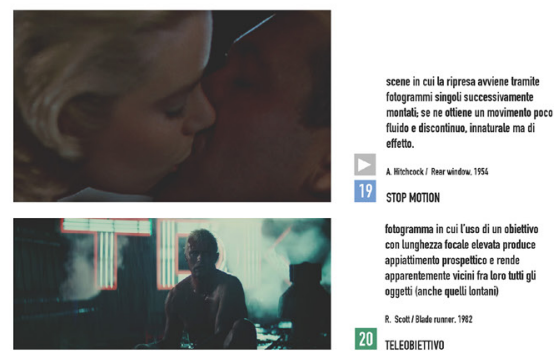

19 STrP MOTOK
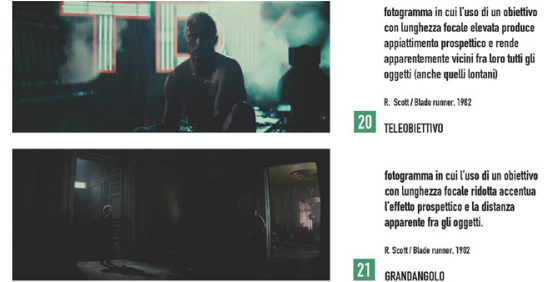

Iologramna in cuil lusodili notiettio

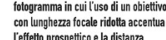
apparenter fra gi ogygeti

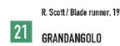

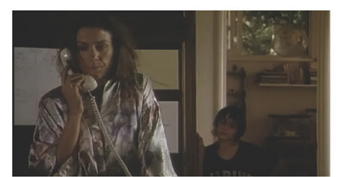

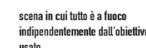

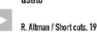

22 PAMFocius
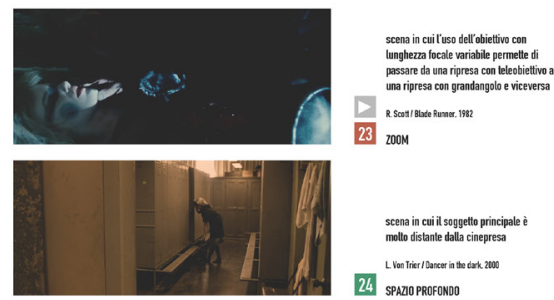

232000

Scena in cuili soggetlo principale

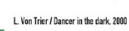

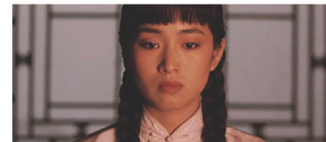

24 SPazlo profono
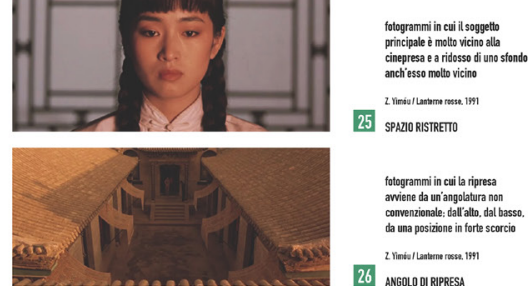

25 SPRlo RISTRETI

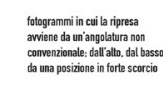

26 ANGollo of RPRESA

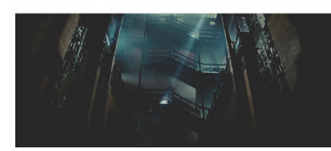

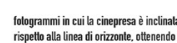

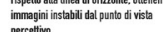

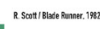

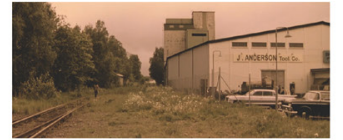

27 RPPESS MCLLNAII

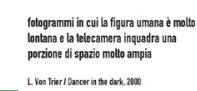

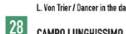

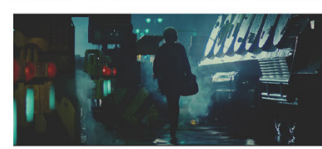

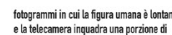

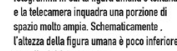

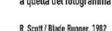

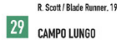

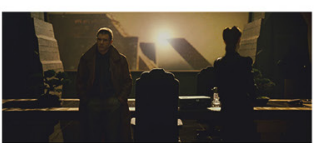

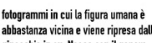

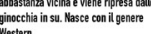

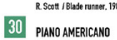

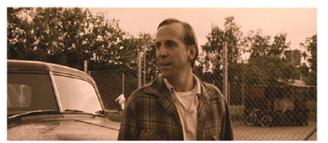

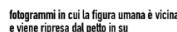

intectat mono

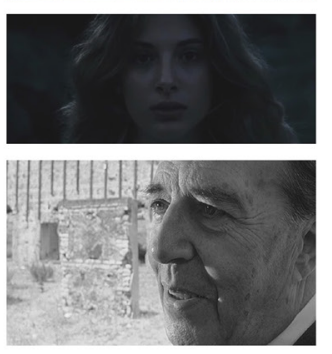

1 Puncuenom

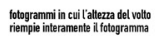

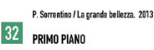

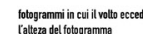

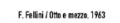

33 PRMMO puMO STRETIO

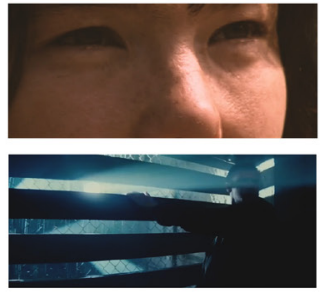

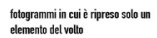

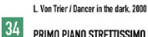

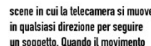
un soggatto. Quando il moviment
del soggetlo e minimo si deffrise
Retraning

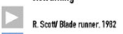

35 RPPESA MOEll.

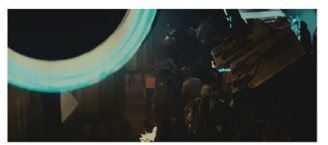

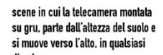

direzione
a

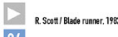

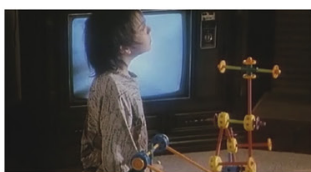

36 CRNES SHOT

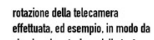

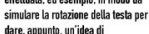

"panorarices"

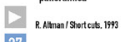

37 PA

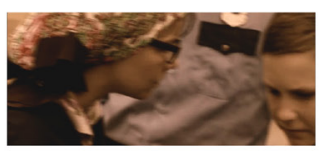

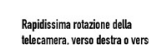

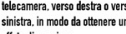

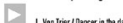

38 WHIP PaN

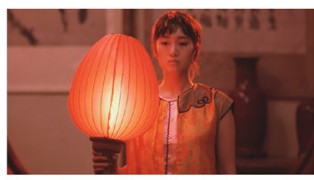

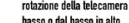

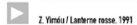

39 TLI

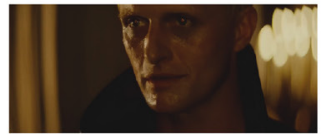

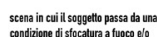

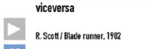

46,97 25,07\% 28, $17 \%$

Fig. 5.Visual qualities:

shooting. 

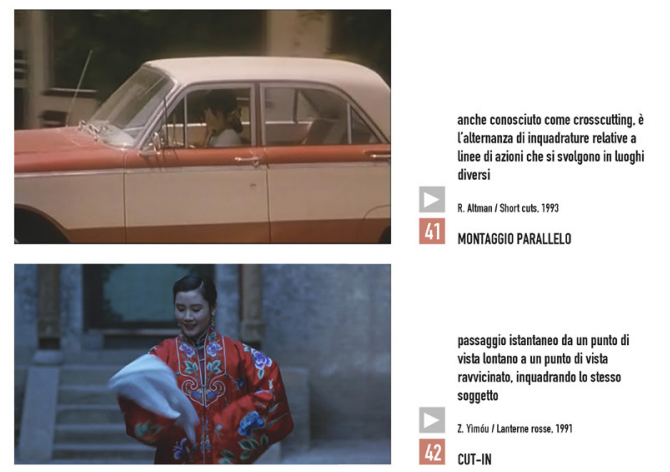

41 montagGio parallelo

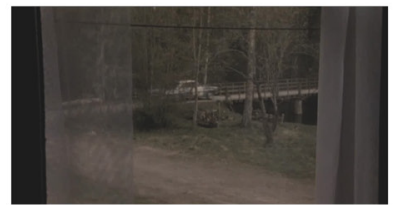

è linverso del cut-in 43 L Von Trier/ Dancer in the dark. 2000
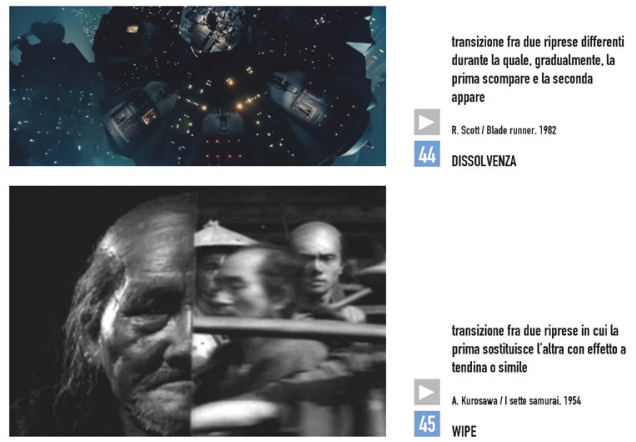

44 R. Sotssollatereruma

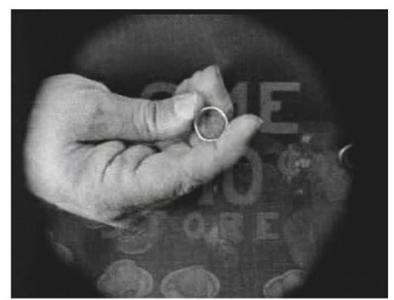

45 WIPE

Fig. 6.Visual qualities: editing.
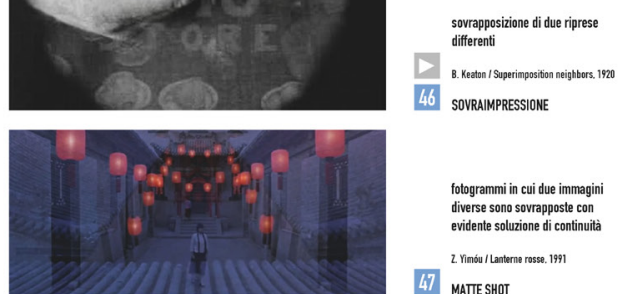

46 SOVRAMPPESSIONE

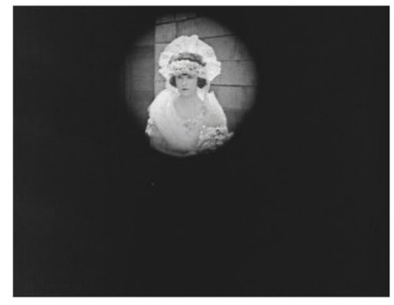

maschera circolare che si alargag (iris-in) o sirestringe (iris-outt sil
scena B. keet 48 IRIS

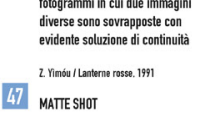
prima sositiuisce laturra con effet

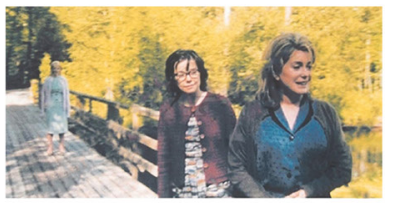

taglio della parte centrale di una ripresa
in modo da collegare bruscamente la parte iniziale e quella finale. Gli oggetti

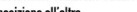

L. Von Tier / Danceri it the dark. 200 49 JUMP-CUT

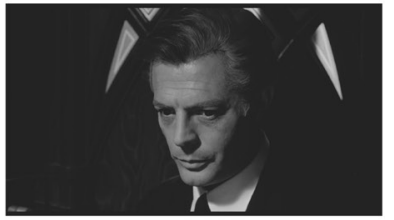

scene in cuil ta telecamera si posiziona dal sognetto inpuadrato precedertemene
F. Fellinil totoe merzo. 1960

50 RIPRESA SOGGETIVA

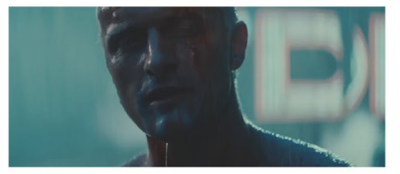

scene che riprendono un soggetto

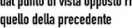
quello della precese
innuadratura

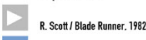

51 CoNrRocampo

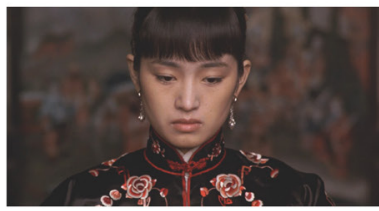

scene che riprendono oli occhidi iun
soggetto e immediatamente dopo ció soggetto e immediatmente do
che il soggetto sta guardando

2. Tinou/lantern rosse. 1991

52 ErelNEMAтCH

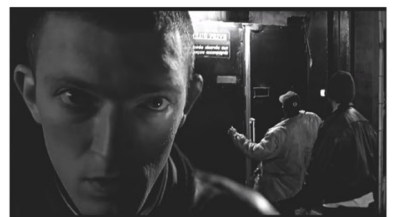

sourapposisione di idue inquadrature diverse ma simili dal punto divista
puramente grafico o melatorice (1) M Kassovit/La haine. 1995

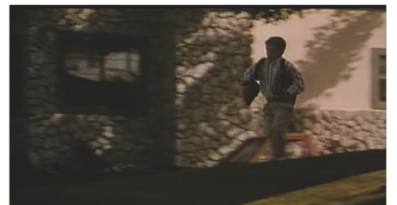

53 GRAPHIC MATCH

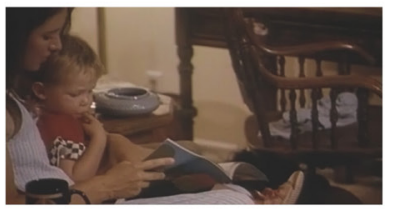
riprendono un soggetto in moviment R. R. Alman I Start cuts. 1999 54 MarCH ON ACTION

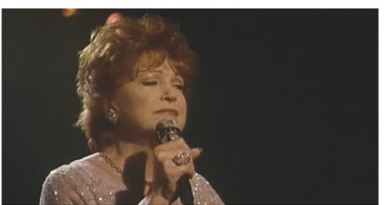

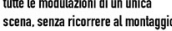
D. Althas / Short ctut. 1999 55 PIANo SEeuerza

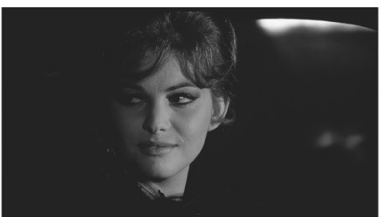

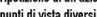
R. Altman / Sthortcuts. 199 56 OVERLAPPING scene in cuile niprese dei personaggi sisommano a unno stondo
un momentio differente

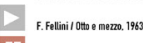

57 REROPPOIEIIONE

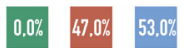


In the specific case of Rear Window we have identified 30 key frames / shots (fig. 7), which obviously cannot make the whole complex visuality of the film but which however can provide useful support to integrate the understanding of the events and reproduce some of the director's choices.

Once the keyframes were identified, we created the $3 \mathrm{~d}$ models of the spaces subject to the shoot. In general, the construction of the $3 \mathrm{~d}$ model can take place in different ways (fig. 8):

- by reconstruction from graphics, if the executive projects of the set are available;

- by in situ surveying, if the shots were taken in accessible public spaces;

- through perspective reconstruction from a single frame, if there are elements of certain dimensions in the shots;

- through reconstruction from stereo photography, if the film has been shot with a stereoscopic camera.

In the experimentation carried out on Rear Window, the construction was done through a single-frame perspective restitution and was favoured by the fact that the shooting of the entire feature film was made exclusively inside a room and on the courtyard of the building on which it overlooks (fig. 9).

Subsequently, we created two maquettes in polylactic acid (PLA) (fig. I0): one relating to the courtyard of the building where the whole story takes place (scale 1: 100) and one relating to the room from which Jeff (the protagonist) observes the events (scale 1:20). Through the preliminary tactile exploration of these two models, before watching the film, the blind
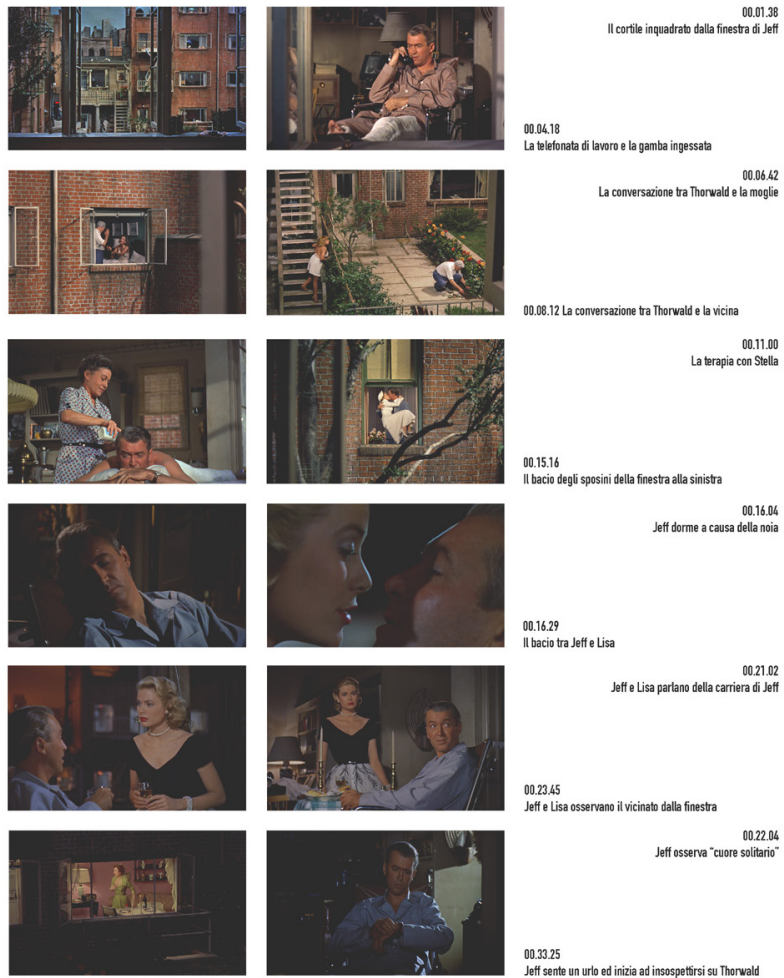

00.23 .45
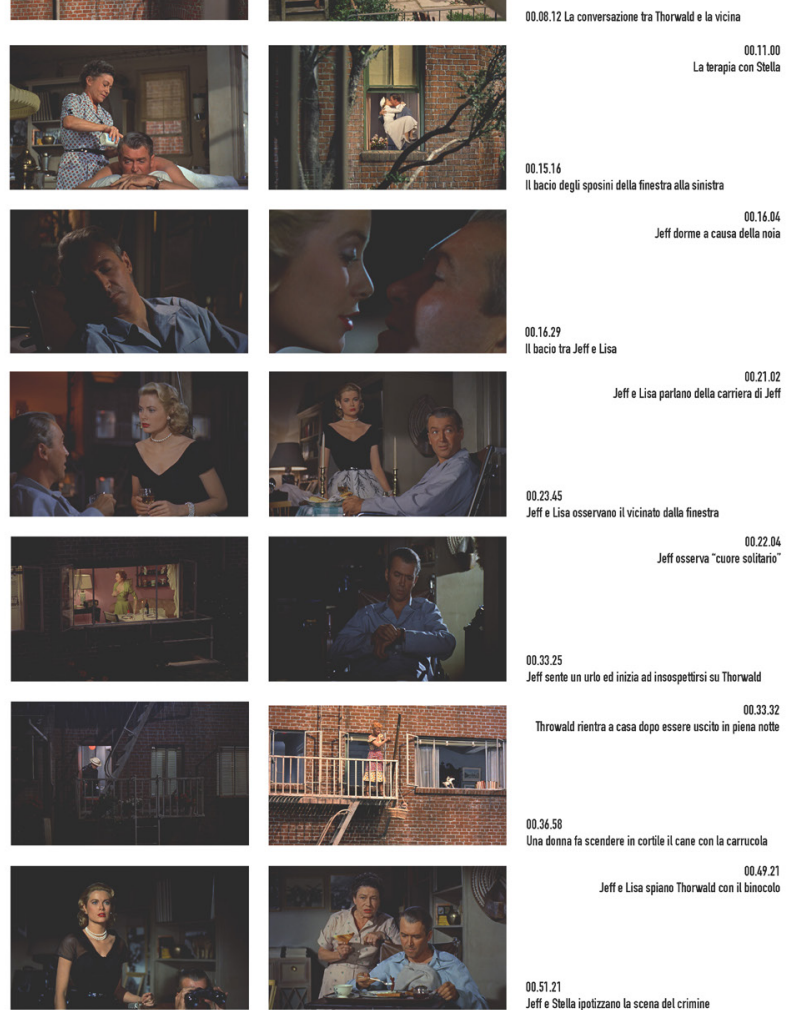

00.33 .32 1.921
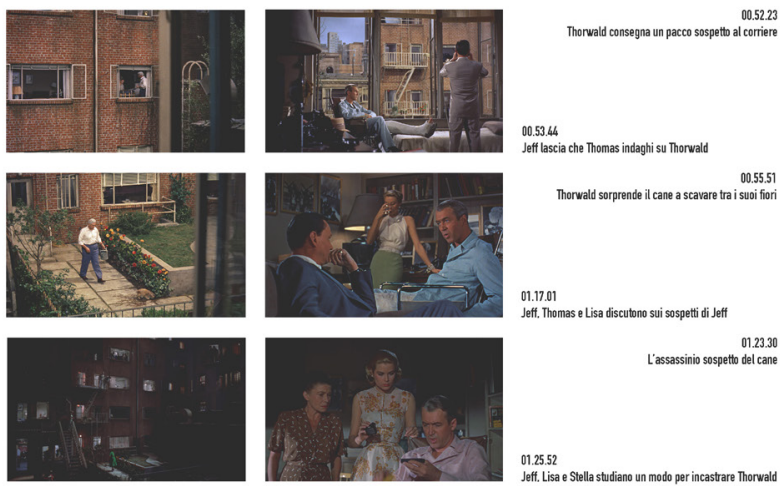
01.17.01
Jeft. Thomas e Lisa dischtonos sui sospetti i i jeff
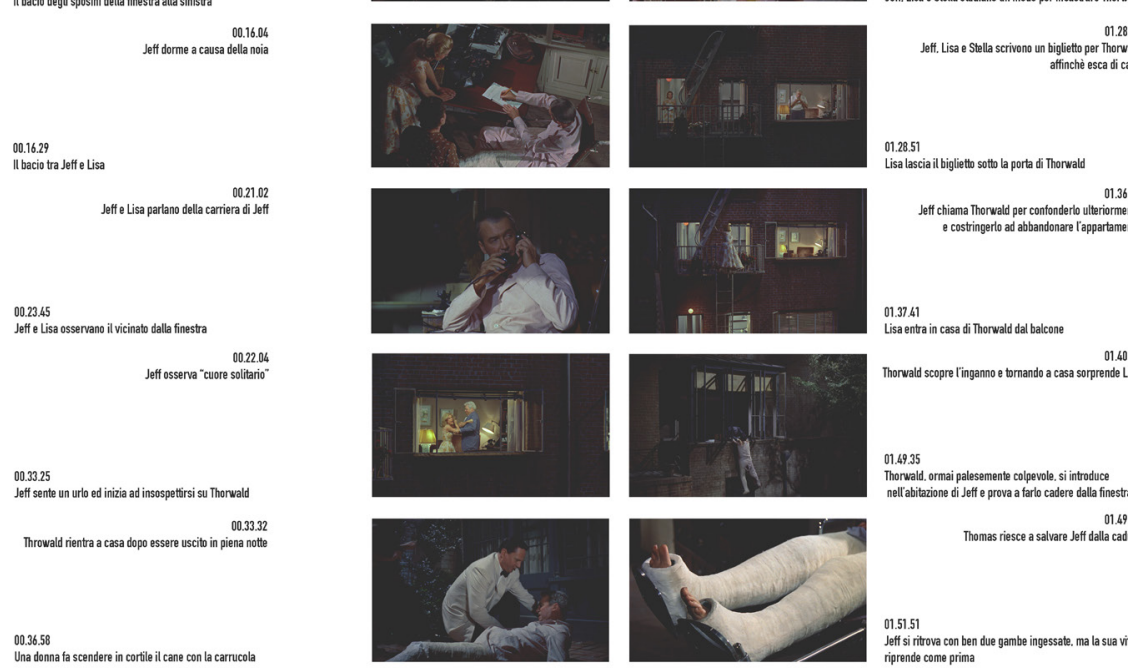

01.4 .935

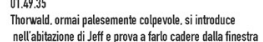

\section{LA COMPOSIZIONE DEL FILM SEQUENZA DI FOTOGRAMMI CHIAVE}


person will be able to get an idea of the characteristics of the space in which the events will take place.

Lastly, the bas-relief of the keyframes that can be rendered via static support were also made in PLA; the criterion was to synthesize the scene through 5 overlapping layers, each of which is included in a flat surface protruding I $\mathrm{mm}$ from the one below (fig. I I). The bas-relief includes a caption in Braille with useful indications to place the scene in the plot of the film (fig. 12).

\section{Final considerations}

The results of the research summarized in this study make it possible to carry out the preparatory work for the construction of the bas-reliefs meant to describe the frames and key scenes of a static film without special effects such as Rear Window. In the specific case, we chose 30 frames which we subsequently transformed into bas-reliefs. A procedure of this type has limits that force it to remain at an experimental level: among the main limits, the amount of bas-reliefs to be created, the consequent cost, the need for additional audio support to allow the blind person to pick up the bas-relief corresponding to the scene at the exact moment, the impossibility of reproducing many of the qualities related to filming (such as camera movements) and all the choices related to editing. Many of these limits will be exceeded as soon as the motorized microbubble tablet is available; with this device, the entire sequence of images of a film can be translated into a tactile support that updates in real time during the projection. But even in this case, an exception must be made for some qualities (related to colours, the use of lights, blurring and intentional blur, etc.) for which the surrogate of the bas-reliefs is not suitable and it is necessary to carry out tests directly on the motorized microbubble tablet. Similar difficulties arise for shooting or editing effects that

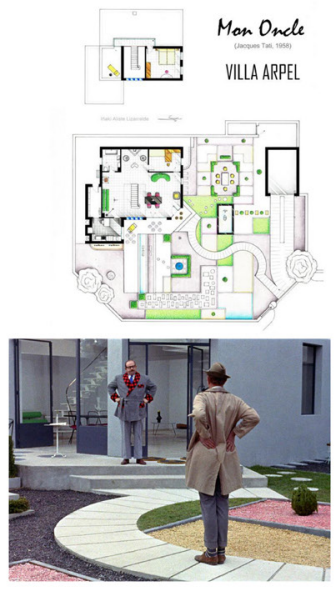

Viene effettuata tramite itridisegno degli elaborat

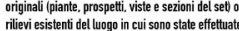
rileviesis
riprese J. Tati/Mon nende. 1958

01 RICOSTRULONe Da Elaboran GraACl

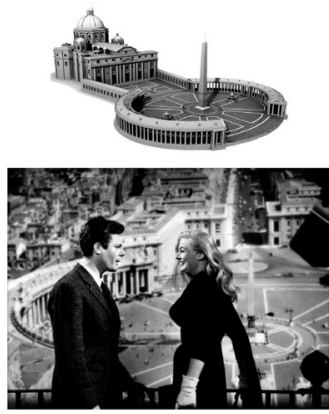

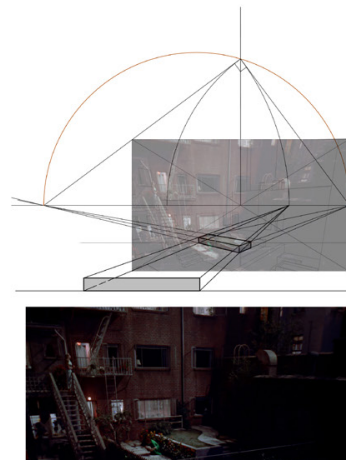
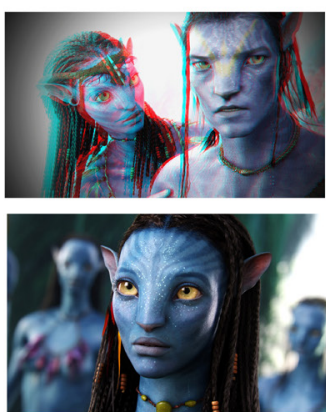

II fotoggramma deve inguadrare la scena in modo che it

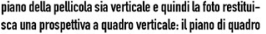

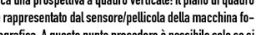

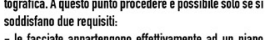

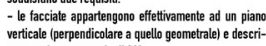
verticale (perpendicolare a qustor

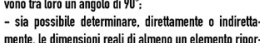
mente, le dime
tato nella foto.

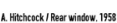

O3 RESTTUZIONE PROSPETICA DA FTTOGRAMM SINGOLO

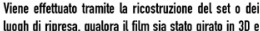

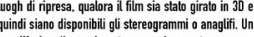
maga, che e un in immagine stereascosopica, o steresegram-

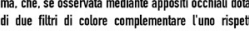

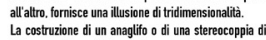

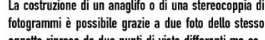

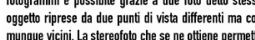
munque vicini. La stereafotot che se ne ettiene perm
di diterminare le misure degli oggeti ipresi. J. Camerern/hwarer. 2009

04 RICOSTRUZIONE DA STEREOCOPPIE 
Fig. 9. Graphic rendering of the room and buildin

where Rear Window is
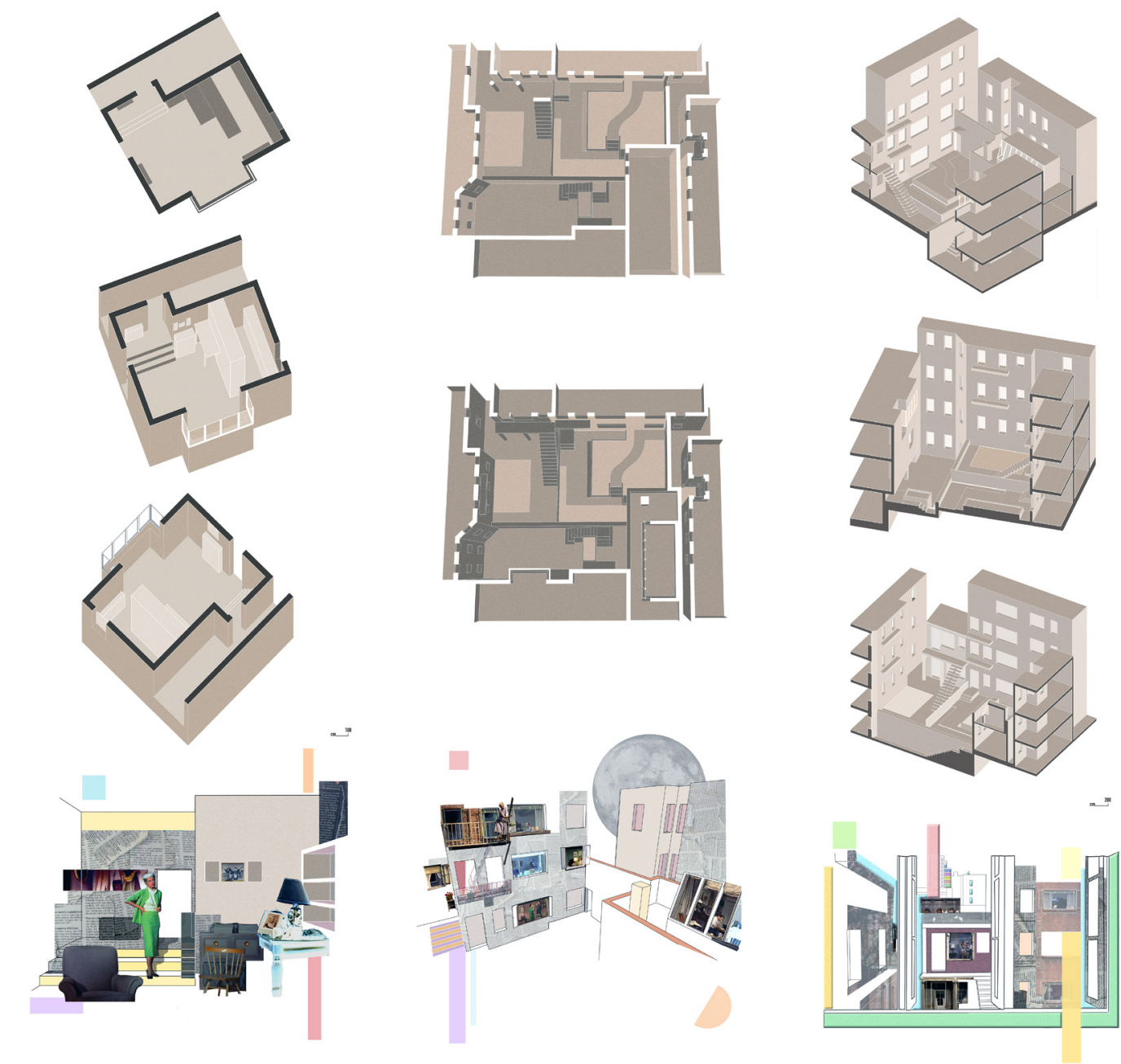

Fig. 10. Views of the

three-dimensional mode of the building in which

Rear Window is set and

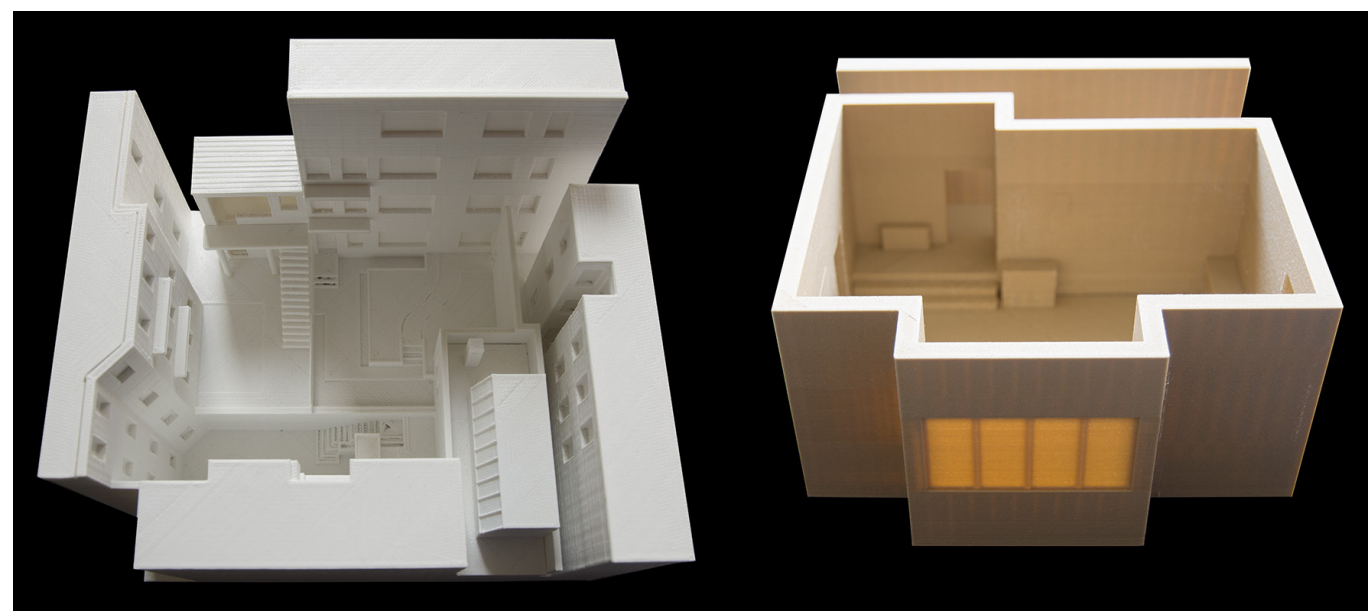




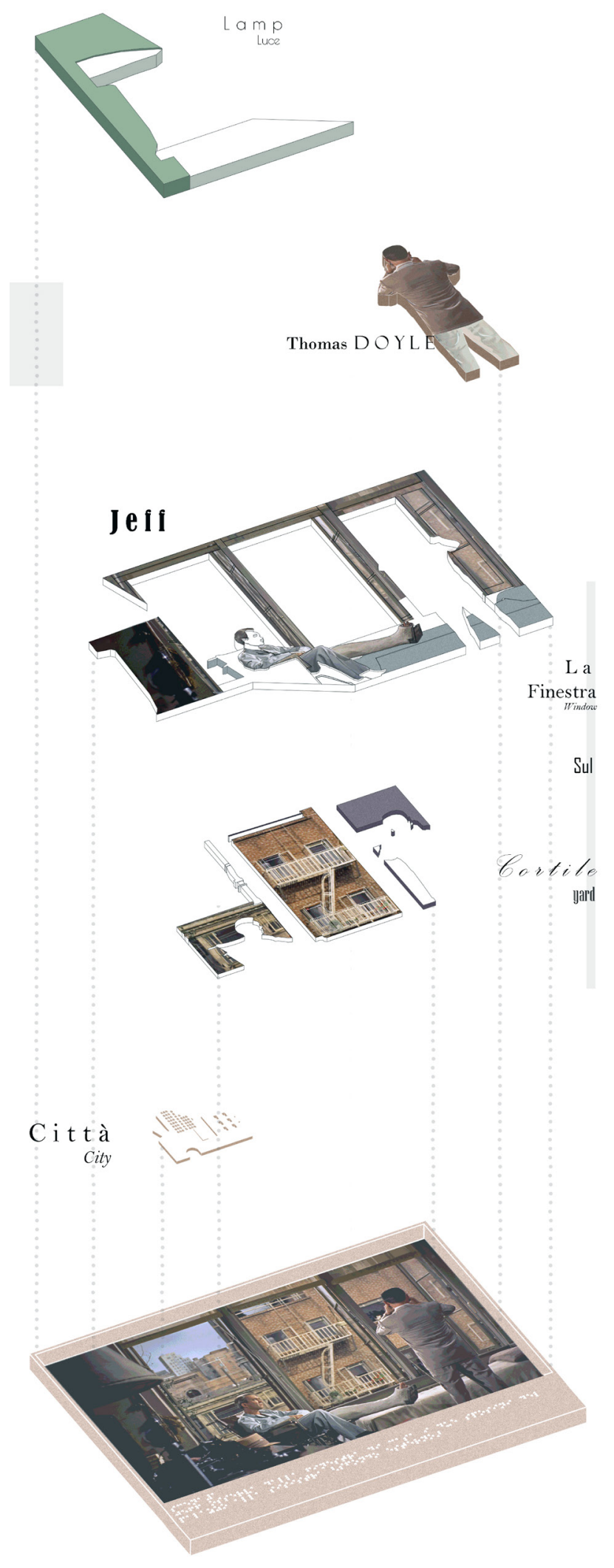


develop in very short times, such as the whip pan. The current limit, therefore, is linked to the unavailability of a support which, as is presumed, will soon be marketed and may offer a new development to ongoing research [7].
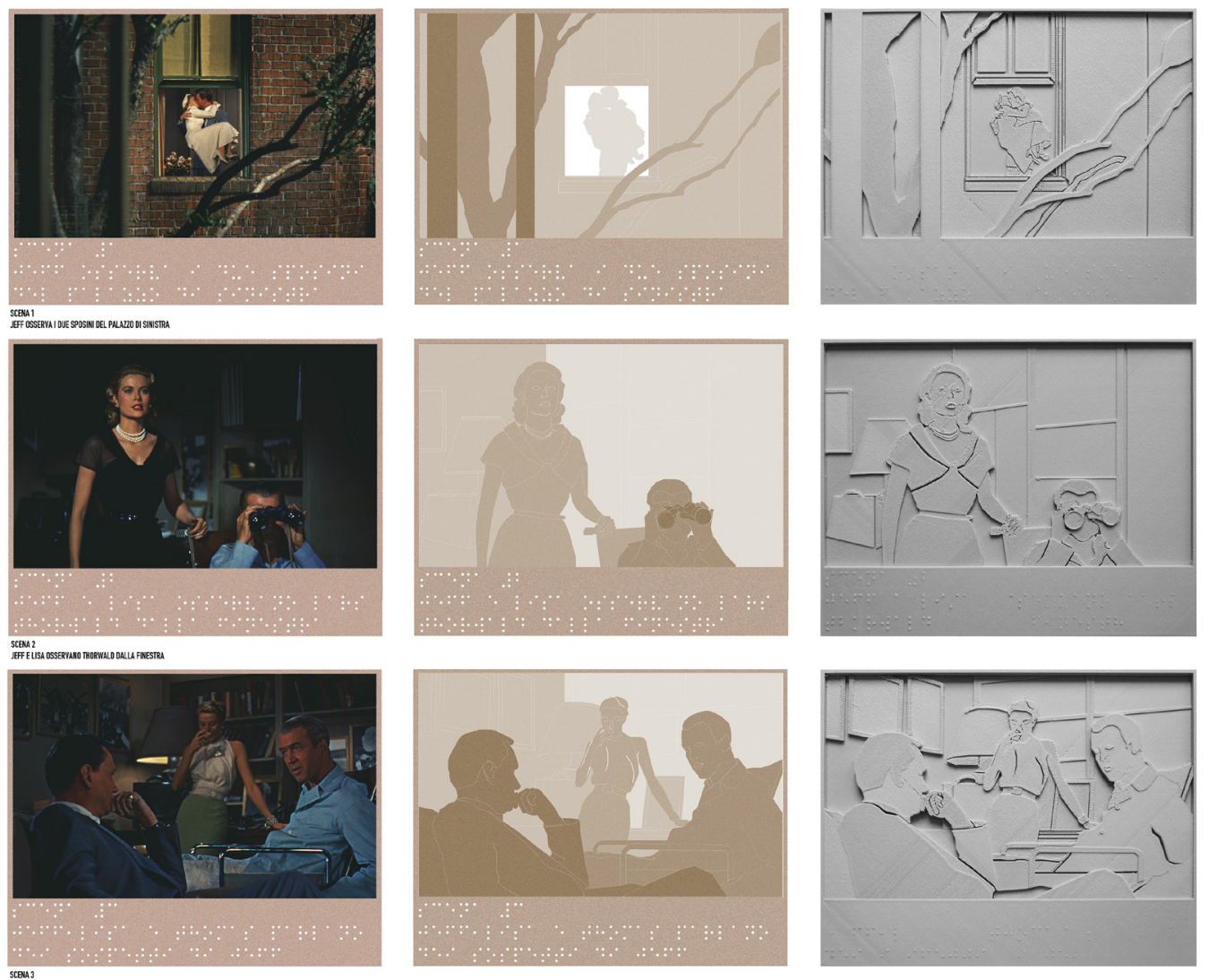

Fig. 12. From frame to the subdivision in layers up to the bas-relief.
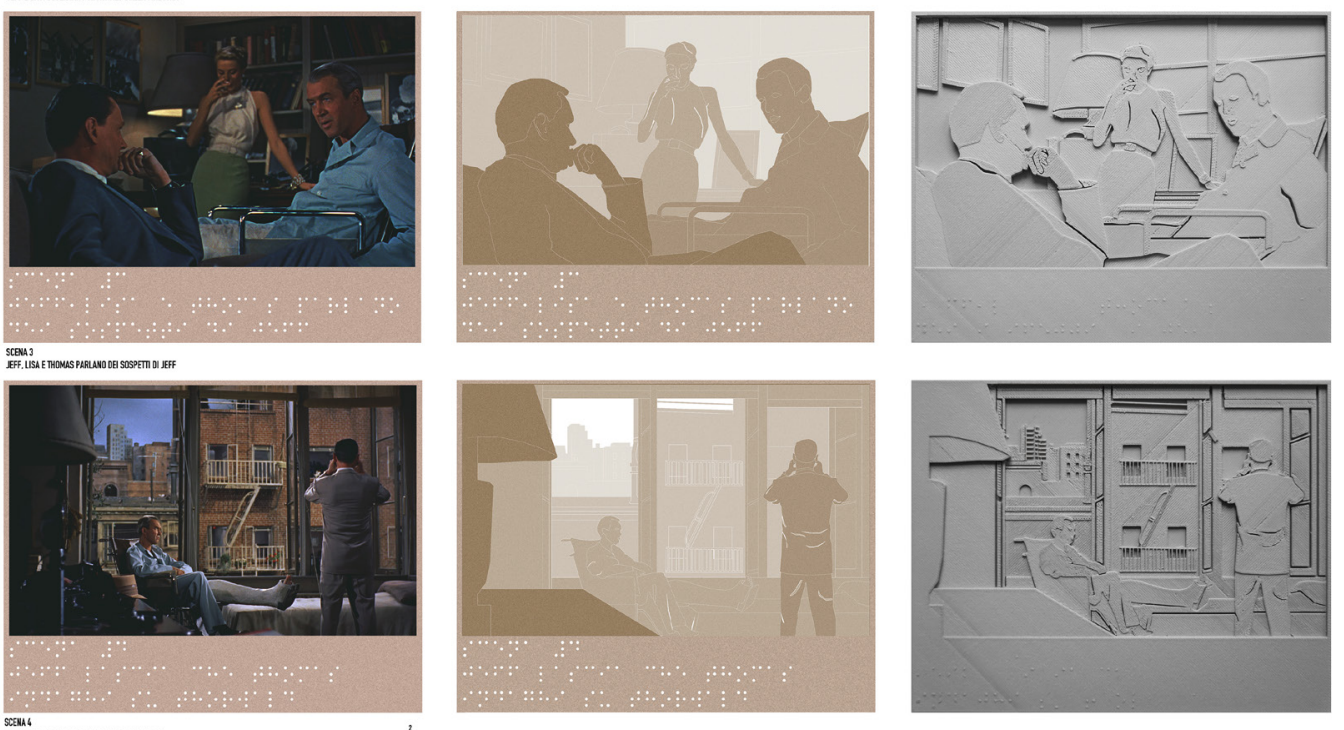

\section{Notes}

[I] On this topic see: Brambring 2006.

[2] The audio description media available can be grouped into two categories: online audio libraries (such as cineaudioteca.it or moviesfortheblind.com) and apps for mobile devices (such as Moviereading or Greta). In both cases, these are supports that help the understanding of what is projected on the screen through a verbal description that, in real time, overlaps the dialogues and sounds of the original film.

[3] On this topic, the bibliography and the experiences carried out are numerous and it is not possible to quote them all here. A valid reference on graphics, especially for pedagogy, is the work by Edman, 1992. Also very interesting are the experiments in which the visually impaired are the ones who make the photographic shots; the most innovative experiences are available on

$<3$ dphotoworks.com> and <blindwithcamera.org>. 
[4]The electromechanical devices suitable for reproducing Braille writing have existed for several decades but have had almost no further development since the reproduction of a text through a grid of points is a very simple operation. The same system (grid of metal or plastic punches that can be raised mechanically) has also been used to reproduce rudimentary shapes, and there are different products on the market (for the texts: <blitab.com $>$ for the elementary shapes: <feelif.com/ blindpad.eu/> or the very recent Tactile Pro <powerct $\mathrm{kr} />$ ). The limit of all these devices is that the grid is in any case constrained to an interaxis between the points that cannot go down to a certain value, and therefore the reproducible forms are very schematic. A few months ago a new tablet has been announced based no longer on punches but on 10,000 motorized points (micro-bubbles placed under an elastic sheath), thus capable of returning much more complex and articulated forms and above all the movement of the forms themselves; at the moment, however, the product has not yet been marketed.

[5] On the theme of perspective, it is necessary to remember the position of Erwin Panofsky and that opposed to it, see: Panofsky 1927; Gioseffi 1957

[6] The work is inspired by an experience, fully reworked, developed at the University of Yale: <filmanalysis.coursepress.yale.edu> For aspects relating to film in general, see: Rondolino, Tomasi 2018; for editing, the main reference is: Cassani 2013.

[7] Daniele Colistra set up the research and wrote the text; Giada Puccinelli carried out the checks of the tactile models, the visual analysis of the films and the images.

\section{References}

Brambring Michael (a cura di). (2006). Early Intervention with Infants and Preschoolers who are Blind. Würzburg: Bentheim.

Cassani Diego (20 I 3). Manuale del montaggio. Tecnica dell'Editing nella comunicazione cinematografica e audiovisiva. Novara: UTET Università.

Edman Polly K. (1992). Tactile Graphics. New York: AFB Press.

EmplerTommaso, Fusinetti Alexandra (2019). Rappresentazione visuo-tattile. Comunicazione tattile per i disabili visivi. In Belard Paolo (a cura di). Riflessioni. L'arte del disegno/il disegno dell'arte. Atti del $41^{\circ}$ Convegno Internazionale dei Docenti delle Discipline della Rappresentazione, Perugia 19-2 I settembre 2019. Roma: Gangemi, pp. I563-I 572

Gioseffi Decio (1957). Perspectiva artificialis. Per la storia della prospettiva. Spigolature e appunti. Trieste: Università degli Studi di Trieste.

Grassini Aldo (20 I 6). Per un'estetica della tattilità. Ma esistono dawero le arti visive? Roma: Armando Editore.

Grassini Aldo, Sòcrati Andrea, Trasatti Annalisa (20 I8). L'arte contemporanea e la scoperta dei valori della tattilità. Roma:Armando Editore.

Levi Fabio, Rolli Rocco (1994). Disegnare per le mani. Manuale di disegno in rilievo. Torino: Zamorani.

Munari Bruno (1986). I laboratori tattili. Bologna: Zanichelli.

Panofsky Erwin (1927). Die Perspektive als "Symbolische Form". Leipzig-Berlin:Teubner. (Trad. it. La prospettiva come "forma simbolica" e altri scritti (1961). Milano: Feltrinelli.

Rondolino Gianni,Tomasi Dario (20 I 8). Manuale del film. Racconto, linguaggio, analisi. Novara: UTET Università.

Sacchetti Anna Maria (2005). Vedere con le mani. Marina di Massa: Edizioni Clandestine.

\section{Authors}

Daniele Colistra, Università degli Studi Mediterranea di Reggio Calabria, daniele.colistra@unirc.it Giada Puccinelli, Università degli Studi Mediterranea di Reggio Calabria, giadapuccinelli@libero.it

To cite this chapter. Colistra Daniele, Puccinelli Giada (2020). Cinema per i non vedenti. Dispositivi tattili per la fruizione/Cinema for the blind Tactile devices for enjoyment. In Arena A., Arena M., Brandolino R.G., Colistra D., Ginex G., Mediati D., Nucifora S., Raffa P. (a cura di). Connettere. Un disegno per annodare e tessere. Atti del $42^{\circ}$ Convegno Internazionale dei Docenti delle Discipline della Rappresentazione/Connecting. Drawing for weaving relationships. Proceedings of the 42th International Conference of Representation Disciplines Teachers. Milano: FrancoAngeli, pp. 31 29-3154. 